\title{
Patient-reported outcomes with durvalumab by PD-L1 expression and prior chemoradiotherapy-related variables in unresectable stage III non-small-cell lung cancer
}

\author{
Marina C Garassino*,1 (D), Luis Paz-Ares², Rina Hui ${ }^{3}$, Corinne Faivre-Finn ${ }^{4}$, Alex Spira ${ }^{5}$, \\ David Planchard $^{6}$, Mustafa Özgüroğlu7 ${ }^{7}$, Davey Daniel $^{8}$, David Vicente ${ }^{9}$, Shuji Murakami ${ }^{10}$, \\ Corey Langer ${ }^{11}$, Suresh Senan ${ }^{12}$, David Spigel ${ }^{13}$, Anna Rydén ${ }^{14}$, Yiduo Zhang ${ }^{15}$, Cathy \\ $\mathrm{O}^{\prime}$ Brien $^{16}$, Phillip A Dennis ${ }^{15}$ \& Scott J Antonia ${ }^{17}$ \\ ${ }^{1}$ Fondazione IRCCS Istituto Nazionale dei Tumori, Milan, 20133, Italy \\ ${ }^{2}$ Hospital Universitario 12 de Octubre, CiberOnc, Universidad Complutense \& CNIO, Madrid, 28041, Spain \\ ${ }^{3}$ Westmead Hospital \& the University of Sydney, Sydney, NSW, 2145, Australia \\ ${ }^{4}$ The University of Manchester \& The Christie NHS Foundation Trust, Manchester, M20 4BX, UK \\ ${ }^{5}$ Virginia Cancer Specialists Research Institute, Fairfax, VA, \& US Oncology Research, The Woodlands, TX 22031, USA \\ ${ }^{6}$ Institut Gustave Roussy, Department of Medical Oncology, Thoracic Group, Villejuif, 94805, France \\ ${ }^{7}$ Istanbul University - Cerrahpaşa, Cerrahpaşa School of Medicine, Istanbul, 34320, Turkey \\ ${ }^{8}$ Sarah Cannon Research Institute/Tennessee Oncology, Chattanooga, TN 37203, USA \\ ${ }^{9}$ Hospital Universitario Virgen Macarena, Seville, 41009, Spain \\ ${ }^{10}$ Kanagawa Cancer Center, Yokohama, 241-8515, Japan \\ ${ }^{11}$ Abramson Cancer Center, University of Pennsylvania, Philadelphia, PA 19104, USA \\ ${ }^{12}$ Department of Radiation Oncology, Amsterdam University Medical Centers, Vrije Universiteit Amsterdam, Amsterdam, \\ 1081, The Netherlands \\ ${ }^{13}$ Sarah Cannon Research Institute/Tennessee Oncology, Nashville, TN 3720231, USA \\ ${ }^{14}$ AstraZeneca, Gothenburg, 431 50, Sweden \\ ${ }^{15}$ AstraZeneca, Gaithersburg, MD 20878, USA \\ ${ }^{16}$ AstraZeneca, Cambridge, CB4 OWG, UK \\ ${ }^{17} \mathrm{H}$. Lee Moffitt Cancer Center \& Research Institute, Tampa, FL 33612, USA \\ *Author for correspondence: Tel.: +39 223903 813; marina.garassino@istitutotumori.mi.it
}

\begin{abstract}
Aim: We retrospectively investigated the impact of tumor PD-L1 expression and prior chemoradiotherapy (CRT)-related variables on patient-reported outcomes (PROs) from PACIFIC. Patients \& methods: PACIFIC was a Phase III study of durvalumab versus placebo after CRT in patients with unresectable, stage III nonsmall-cell lung cancer. If available, pre-CRT tumor tissue was tested for PD-L1 tumor-cell expression, scored at prespecified (25\%) and post-hoc (1\%) cut-offs. PROs were assessed using EORTC QLQ C30/-LC13. Results: Similar to the intent-to-treat (ITT) population, most PROs remained stable over time across PD-L1 and CRT subgroups, with few clinically relevant differences between treatment arms. Time to deterioration was generally similar to the ITT population. Conclusion: Neither PD-L1 expression nor prior CRT-related variables influenced PROs with durvalumab therapy.
\end{abstract}

Clinical trial registration: NCT02125461 (ClinicalTrials.gov)

Lay abstract: Durvalumab works with the immune system to find and attack certain types of cancer by binding to and blocking a protein called PD-L1. Based on the placebo-controlled, Phase III PACIFIC study, durvalumab is approved for the treatment of adults with stage III, non-small-cell lung cancer whose disease cannot be removed by surgery and has not progressed after platinum-based chemoradiotherapy (CRT). Although enrolment in PACIFIC was not restricted by tumor PD-L1 expression, we investigated whether it had any impact on patient-reported outcomes (including symptoms, functioning and global health status or quality of life), as assessed using questionnaires administered during the study. We also investigated whether different features of prior CRT had any impact on such outcomes. In summary, durvalumab, 
compared with placebo, had no detrimental effect on patient-reported outcomes, regardless of PD-L1 expression or features of prior CRT.

First draft submitted: 29 October 2020; Accepted for publication: 20 November 2020; Published online: 15 February 2021

Keywords: durvalumab • patient-reported outcomes $\bullet$ programmed cell death ligand-1

Platinum-based concurrent chemoradiotherapy (CRT) has historically been the standard of care for patients with unresectable, stage III non-small-cell lung cancer (NSCLC) [1]; however, long-term outcomes were poor (5-year survival rates 15-32\%) [2,3]. Furthermore, survival outcomes are not improved when patients with stage III NSCLC are treated with chemotherapy (CT) following CRT, underscoring a prior unmet need for effective consolidative therapies [4].

In the Phase III PACIFIC study (ClinicalTrials.gov: NCT02125461) of patients with unresectable, stage III NSCLC without disease progression after platinum-based CRT [5], the anti-PD-L1 antibody durvalumab significantly improved progression-free survival (PFS; hazard ratio [HR]: 0.52, 95\% CI: 0.42-0.65; p < 0.0001; median 16.8 vs 5.6 months) and overall survival (OS; HR: 0.68; 95\% CI: 0.53-0.87; $\mathrm{p}=0.00251$; median not reached vs 28.7 months) compared with placebo, and durvalumab exhibited a manageable safety profile [6,7]. These data, which were recently reinforced by updated survival analyses at 4 years, demonstrating consistent and durable benefit with durvalumab [8], established the 'PACIFIC regimen' (12 months durvalumab following platinum-based CRT) as the current standard of care in unresectable, stage III NSCLC, with global approvals received [7,9].

There is increasing interest in the collection of patient-reported outcomes (PROs) [10-12]. In the PACIFIC trial, a prespecified analysis of PROs as a secondary end point indicated that treatment with durvalumab did not compromise patients' symptoms, functioning, or global health status/quality of life (QoL) compared with placebo in the intent-to-treat (ITT) population [13].

PD-L1 expression on tumor cells (TCs) has been identified as a positive predictive biomarker for the efficacy of PD-1/PD-L1 immune checkpoint inhibitors (ICIs), with treatment guidelines mandating PD-L1 positivity (at varying cut-offs) for the use of several of these agents; however, meta-analysis data indicate that PD-L1 expression is an imperfect predictive biomarker for OS benefit with PD-1/PD-L1 ICI therapy [14]. Furthermore, data from previous Phase III trials of NSCLC demonstrate that, although PD-L1 positivity can be used to enrich the population with higher response rates, a proportion of patients with PD-L1 negative tumors can still display durable benefit $[15-17]$.

Patients were enrolled into the PACIFIC trial irrespective of PD-L1 status; PACIFIC was designed to assess PFS and OS in a broad population, but included an exploratory analysis based on a prespecified PD-L1 expression threshold of TC 25\% (assessed in archived, pre-CRT tumor samples, where available). However, in response to a request from the European Medicines Agency (EMA), an additional exploratory analysis with a post-hoc PD-L1 expression threshold of TC $1 \%$ was also conducted $[6,18]$. While durvalumab provided PFS benefit compared with placebo irrespective of tumoral PD-L1 expression, OS benefit could not be definitively demonstrated in the relatively small subgroup of patients with PD-L1 TC $<1 \%(n=148)$. Based on these data, the EMA restricted the use of durvalumab to patients with histologically confirmed PD-L1 TC $\geq 1 \%$ [7]. In the context of the EMA regulatory decision and the broader US approval regardless of PD-L1 status, there is a need to better understand the impact, if any, of PD-L1 expression on PROs in patients treated with durvalumab.

In addition to tumoral PD-L1 expression, CRT regimens received prior to or alongside ICI can influence clinical outcomes [19]. Preclinical and clinical data indicate that CT and radiotherapy (RT) prime the tumor microenvironment for response to PD-1/PD-L1 ICI, inducing changes to the expression of cell surface and secreted immunomodulatory markers (including upregulation of PD-L1) and to the composition of the local immune cell population [20-28]. Moreover, lingering toxicities from prior CRT may potentially affect safety and PROs with subsequent treatment. Therefore, a better understanding of the impact of variables related to prior CRT (such as the dose and timing of RT, and the specific chemotherapeutic agents utilized) on PROs with durvalumab is required.

Based on the EMA decision to restrict approval of durvalumab to patients with PD-L1 TC $\geq 1 \%$, the primary focus of this analysis was to better understand the impact of PD-L1 expression on the benefit/risk profile of durvalumab. We carried out exploratory, post-hoc analyses of PROs (symptoms, functioning, or global health 
status/QoL) in patient subgroups defined by the previously reported PD-L1 expression thresholds, incorporating all PROs that were assessed in the ITT population [13]. As a secondary focus, we also analyzed PROs in patient subgroups defined by variables related to prior CRT, incorporating only PROs that were considered to be of primary interest for the analysis of the ITT population (as treatment benefit was observed with durvalumab irrespective of CRT-related variables) [29].

\section{Patients \& methods}

\section{Study design}

The eligibility criteria and study design for the PACIFIC study, a randomized, double-blind, international, multicenter, Phase III trial, have been described previously [5,6].

Briefly, patients with unresectable, stage III NSCLC and WHO performance status $0 / 1$ who had received $\geq 2$ cycles of platinum-based concurrent CRT were randomized (2:1), within 1-42 days of CRT (within 1-14 days in an earlier protocol prior to amendment), to receive either durvalumab $(10 \mathrm{mg} / \mathrm{kg}$ intravenously; AstraZeneca, DE, USA) or placebo every 2 weeks for $\leq 12$ months. Randomized patients were stratified according to age ( $<65$ vs $\geq 65$ years), sex (male vs female) and smoking history (current/former smoker vs never smoked). Key exclusion criteria included pneumonitis $\geq$ grade 2 or unresolved toxicity $>$ grade 2 (per Common Terminology Criteria for Adverse Events [CTCAE] version 4.03) from prior CRT. Patients provided archived, pre-CRT, tumor tissue samples for PD-L1 testing if available; however, enrolment was not restricted by PD-L1 expression.

Study drug administration commenced after randomization on day 1 and continued until confirmed disease progression, initiation of alternative anticancer therapy, unacceptable toxic effects, or withdrawal of consent. Patients could be treated through progression based on predefined criteria, and could receive the drug again if disease control had been achieved at the end of the 12-month treatment period but disease progressed during follow up (provided they had not received another subsequent systemic anticancer therapy).

All patients provided written informed consent for participation in the study, which was approved by relevant ethics committees and performed in accordance with the International Conference on Harmonisation Guidelines on Good Clinical Practice and the Declaration of Helsinki.

\section{End points \& assessments}

The primary end points were PFS (assessed by blinded independent central review [BICR] according to Response Evaluation Criteria in Solid Tumors [RECIST], version 1.1) and OS. The prespecified secondary end points included the proportion of patients with objective response, duration of response and time to death or distant metastasis (all assessed by BICR [RECIST v1.1]), as well as safety (graded using CTCAE version 4.03) and PROs (symptoms, functioning, or global health status/QoL).

Optional pre-CRT tumor tissue samples were assessed for PD-L1 TC expression using the VENTANA PD-L1 (SP263) immunohistochemistry (IHC) assay (Ventana Medical Systems, Inc., AZ, USA). Testing was carried out at a central laboratory by pathologists trained and qualified by Ventana to score the samples at validated prespecified (25\%) and post-hoc (1\%) thresholds. Patients for whom a tumor tissue sample was not provided, or for whom the provided sample was inadequate for histological evaluation, were classified as having unknown PD-L1 status.

Separately, patients were also divided into subgroups according to variables related to CRT received prior to durvalumab or placebo, including: receipt of pre-CRT induction CT (yes/no); platinum CT agent utilized (cisplatin/carboplatin); receipt of taxane-based CT (yes/no); total radiation dose received ( $<60$ gray $[\mathrm{Gy}] / 60-66 \mathrm{~Gy} />66 \mathrm{~Gy})$; and time elapsed from completion of RT to randomization to study treatment $(<14$ days $/ \geq 14$ days $)$.

PRO scores for symptoms, functioning and global health status/QoL were assessed using two European Organization for Research and Treatment of Cancer (EORTC) questionnaires, as described previously [13]. The 30-item QoL Questionnaire - Core 30 (EORTC QLQ-C30; version 3) comprises five multi-item functioning scales, one multi-item global health status/QoL scale, three multi-item symptom scales and six single-item measures for dyspnea, loss of appetite, insomnia, constipation, diarrhea and perceived financial difficulties [30]. An additional 13-item module, the QoL Questionnaire - Lung Cancer 13 (EORTC QLQ-LC13), was used to address disease/treatment specific symptoms [31]. EORTC QLQ-LC13 comprises one multi-item dyspnea scale and single-item measures for lung cancer-associated symptoms and side effects from conventional CRT. Lung cancer-associated symptoms were the primary focus of this analysis. 
Paper questionnaires were used to assess PROs at week 4, week 8, then every 8 weeks until week 48, then every 12 weeks until disease progression. For patients who discontinued treatment with durvalumab or placebo due to progression, the last assessment was conducted 30 days after administration of the final dose. Questionnaire completion continued following discontinuation until confirmed disease progression; if (at the investigator's discretion) treatment was continued beyond progression due to clinical benefit, the affected patients continued completing questionnaires as long as they remained on treatment.

\section{Statistical analysis}

The sample size used in the PACIFIC trial was calculated to power the study for the primary end points, PFS and OS [5,6]; the assessment of PROs in the ITT population was a prespecified secondary end point, with PRO data summarized and analyzed at the time of the final analysis for the primary end point OS (data cut-off [DCO], 22 March 2018) [6]. Exploratory, post-hoc analyses of PROs were conducted (using the same DCO) in patient subgroups defined by PD-L1 expression (at prespecified and post-hoc PD-L1 TC thresholds) and prior CRT-related variables. Differences in PROs (in terms of mean change in score from baseline [assessed at week 48], time to deterioration [TTD] and improvement rate) following 12 months of either durvalumab or placebo were assessed within each of the defined patient subgroups.

To capture clinically meaningful changes/differences in PROs, scores for symptoms, functioning, or global health status/QoL were calculated according to scoring manual/guidelines and range from $0-100$. Higher scores are indicative of greater severity for symptom scales/items and better health status/function on global health status/QoL and functioning scales. A change in score $\geq 10$ points was considered a clinically meaningful event; specifically, this was a $\geq 10$-point increase for symptom scales/items and a $\geq 10$-point decrease for functioning scales and global health status/QoL [32]. Therefore, EORTC QLQ-C30 global health status/QoL and functional scales were based on patients with baseline scores $\geq 10$; EORTC QLQ-C30 and EORTC QLQ-LC13 symptom scales/items were based on patients with baseline scores $\leq 90$.

For the analysis of change in PRO score at week 48 (from baseline), the identified longitudinal end points of interest in the ITT population were global health status/QoL, physical functioning, fatigue, appetite loss (all EORTC QLQ-C30), cough, dyspnea and chest pain (all EORTC QLQ-LC13). For TTD, these were cough, dyspnea, chest pain, hemoptysis (all EORTC QLQ-LC13) and global health status/QoL (EORTC QLQ-C30); the same end points were included in the improvement rate analysis [13]. Only longitudinal end points considered of primary interest in the ITT population were analyzed for the CRT subgroups. Due to the EMA decision to delineate patient populations indicated for durvalumab therapy by PD-L1 expression, a more in-depth analysis was conducted for the PD-L1 subgroups, incorporating a wider range of symptom scales/items and functioning scales to further define the risk/benefit/risk profile of durvalumab across these populations.

For measures of symptoms, functioning and global health status/QoL, changes in score at week 48 (relative to baseline) were summarized descriptively for the ITT population and all analyzed subgroups.

For all subgroups, TTD was analyzed using the criteria employed for a prespecified analysis of TTD in the ITT population (where TTD was defined as the time from randomization until the date of the first clinically meaningful deterioration/worsening $[\geq 10$ points increase in score for symptoms or $\geq 10$ points decrease in score for functioning and global health status/QoL] or death, irrespective of patient withdrawal from the study treatment or receipt of another anticancer therapy prior to deterioration) [13]. HRs and 95\% CIs were estimated from a Cox proportional-hazards model using an unstratified approach for the subgroups; for the ITT population, the analysis was stratified by age at randomization $(<65$ vs $\geq 65$ ), sex (male vs female) and smoking history (current/former smoker vs non smoker) [13]. For the PD-L1 subgroups only, an additional analysis of TTD was carried out, in which the PRO scores were evaluated at the next consecutive time point following the first observation of a clinically relevant observation (based on a post-hoc analysis of TTD carried out in the ITT population) [13]. In this additional analysis, patients were considered to have experienced deterioration only if the next consecutive observation was also a clinically relevant deterioration from baseline; this was to reduce potential bias by identifying transient deteriorations in PROs (e.g. those associated with temporary treatment side effects/co-morbidities) which may have confounded symptom change associated with disease progression. The statistical methods used were identical to the above-mentioned subgroup analysis of TTD.

PRO improvement rates within all subgroups were calculated using the criteria prespecified for the analysis of improvement rate in the ITT population [13]. The improvement rate for PROs was defined as the percentage of patients with two consecutive assessments, $\geq 14$ days apart, which showed a clinically meaningful improvement from 
baseline; this was taken as a $\geq 10$-point decrease in score for symptoms and $\mathrm{a} \geq 10$-point increase for functioning and global health status/QoL. Odds ratios (ORs) and 95\% CIs were calculated using logistic regression; for the ITT population this was adjusted according to study treatment, age at randomization ( $<65$ vs $\geq 65$ years), sex (male vs female) and smoking history (current/former smoker vs non-smoker) [13]. However, for the PD-L1 and CRT subgroups this analysis was conducted using an unstratified approach. No adjustments were made for multiplicity among any of the subgroup analyses.

All analyses were conducted using SAS ${ }^{\circledR}$ version 9.4. The PACIFIC study is registered with ClinicalTrials.gov (NCT02125461) and EudraCT (2014-000336-42).

\section{Results}

\section{Patients \& PD-L1 expression}

PROs for the ITT population have been reported previously [13]. In total, 709 of 713 patients who were randomized between 9 May 2014 and 22 April 2016 received study treatment (durvalumab, n/N = 473/476; placebo, $\mathrm{n} / \mathrm{N}=236 / 237$ ); as of 22 March 2018, the median follow-up was 25.2 months (range: 0.2-43.1) [6]. A total of $63 \%$ of all randomized patients (451/713) provided a tissue sample of sufficient quality and quantity to determine tumoral PD-L1 expression levels (Figure 1). The remaining 37\% of patients $(262 / 713)$ were therefore defined as having unknown PD-L1 status. The proportion of patients with unknown PD-L1 status was well balanced between the durvalumab $(37 \% ; 174 / 476)$ and placebo arms $(37 \%$; 88/237), as were the proportions of patients with PDL1 TC $\geq 25 \%$ (durvalumab: 24\% [115/476]; placebo: 19\% [44/237]), <25\% (durvalumab: $39 \%$ [187/476]; placebo: 44\% [105/237]), $\geq 1 \%$ (durvalumab: $45 \%$ [212/476]; placebo: 38\% [91/237]) and $<1 \%$ (durvalumab: $19 \%$ [90/476]; placebo: $24 \%$ [58/237]).

\section{Compliance with EORTC QLQ-C30 \& EORTC QLQ-LC13 from baseline to week 48}

In the ITT population, $>79 \%$ of patients completed both EORTC questionnaires up to week 48 in both treatment arms. Across the PD-L1 subgroups, minimum compliance with EORTC questionnaires up to week 48 was $>73 \%$ in the durvalumab arm and $>69 \%$ in the placebo arm (Supplementary Table 1).

Across the CRT subgroups, minimum compliance with EORTC questionnaires up to week 48 was $>71 \%$ in the durvalumab arm and $>57 \%$ in the placebo arm (Supplementary Table 2).

\section{Changes in PRO scores at week 48 (from baseline)}

PRO scores for most symptoms, functioning and global health status/QoL remained stable at week 48 (from baseline) across all five PD-L1 subgroups (including patients with unknown PD-L1 status), and were generally similar to the ITT population, with no clinically meaningful between-arm differences observed for most scores (Supplementary Table 3). This was true for scores assessed using both the EORTC QLQ-C30 and the lung cancerspecific EORTC QLQ-LC13, including the key longitudinal end points of global health status/QoL, physical functioning, fatigue, appetite loss (all EORTC QLQ-C30), cough, dyspnea and chest pain (all EORTC QLQLC13). Of note, in the durvalumab arm, clinically meaningful improvements from baseline were observed across all but one (TC $\geq 25 \%$ ) of the PD-L1 subgroups for dysphagia (mean changes from baseline, -8.1 to -20.9 ) and across all PD-L1 subgroups for alopecia (mean changes: -15.5 to -26.9) (Figure 2). Clinically meaningful improvements in scores for the symptoms of dysphagia (mean changes: -10.4 to -19.4 ) and alopecia (mean changes: -15.8 to -31.3 ) were also observed across all PD-L1 subgroups in the placebo arm. Within each PD-L1 subgroup, the magnitudes of improvement in these symptoms at week 48 were generally similar between the treatment arms (Supplementary Table 3).

Across the CRT subgroups, scores for global health status/QoL, physical functioning, fatigue, appetite loss (all EORTC QLQ-C30), cough, dyspnea and chest pain (all EORTC QLQ-LC13) were generally stable at week 48 (from baseline), with some limited instances of clinically meaningful between-arm differences (Supplementary Table 4). These differences were among patients who were randomized to study treatment $<14$ days after completing RT (cough [mean change: -1.64 and -11.76 with durvalumab and placebo, respectively] and appetite loss [-4.84 and -19.61, respectively]), and patients who received a total RT dose $<60$ Gy (chest pain [mean change: -10.41 and 0.00 , respectively]) or $>66 \mathrm{~Gy}$ (chest pain [mean change: 0.01 and 11.13 , respectively] and cough [-11.11 and 0.00 , respectively]). 


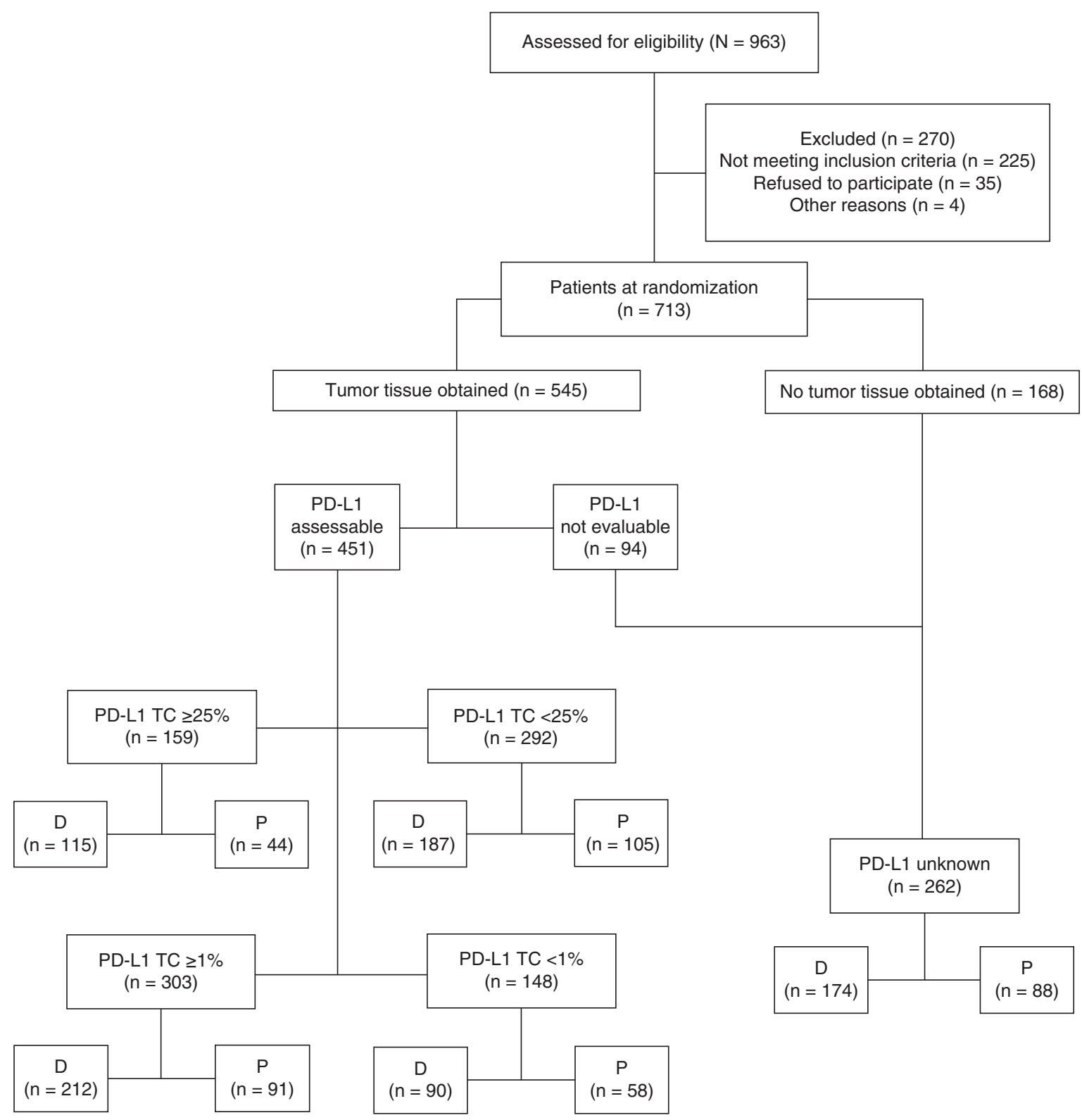

Figure 1. CONSORT diagram (PD-L1 subgroups). The PACIFIC study protocol and amendments were approved by the relevant ethics committees and the study was performed in accordance with the International Conference on Harmonisation Guidelines on Good Clinical Practice and the Declaration of Helsinki. PRO analyses were performed using EORTC QLQ-C30 and EORTC QLQ-LC13 questionnaires. Pre-CRT tumor tissue samples were assessed using the VENTANA PD-L1 (SP263) IHC assay and scored at validated prespecified (TC 25\%) and post-hoc (TC 1\%) thresholds. CRT: Concurrent chemoradiotherapy; D: Durvalumab; EORTC QLQ-C30: European Organisation for Research and Treatment of Cancer Quality of Life Questionnaire - Core 30; EORTC QLQ-LC13: European Organisation for Research and Treatment of Cancer Quality of Life Questionnaire - Lung Cancer 13; IHC: Immunohistochemistry; P: Placebo; TC: Tumor cell.

\section{Time to deterioration}

Across the PD-L1 subgroups, results for TTD of symptoms, functioning and global health status/QoL were generally similar to findings for the ITT population, with treatment effects (HRs) and associated 95\% CIs overlapping between these populations for most PROs (Figure 3). In general, there were no clinically important between-arm differences in TTD of the key symptoms of cough, dyspnea, chest pain, hemoptysis (all EORTC QLQ-LC13) and global health status/QoL (EORTC QLQ-C30). However, TTD of chest pain was longer with placebo, versus durvalumab, among patients with PD-L1 TC $<25 \%$ and patients with PD-L1 TC $<1 \%$, and 
(A)

Dysphagia (LC13)

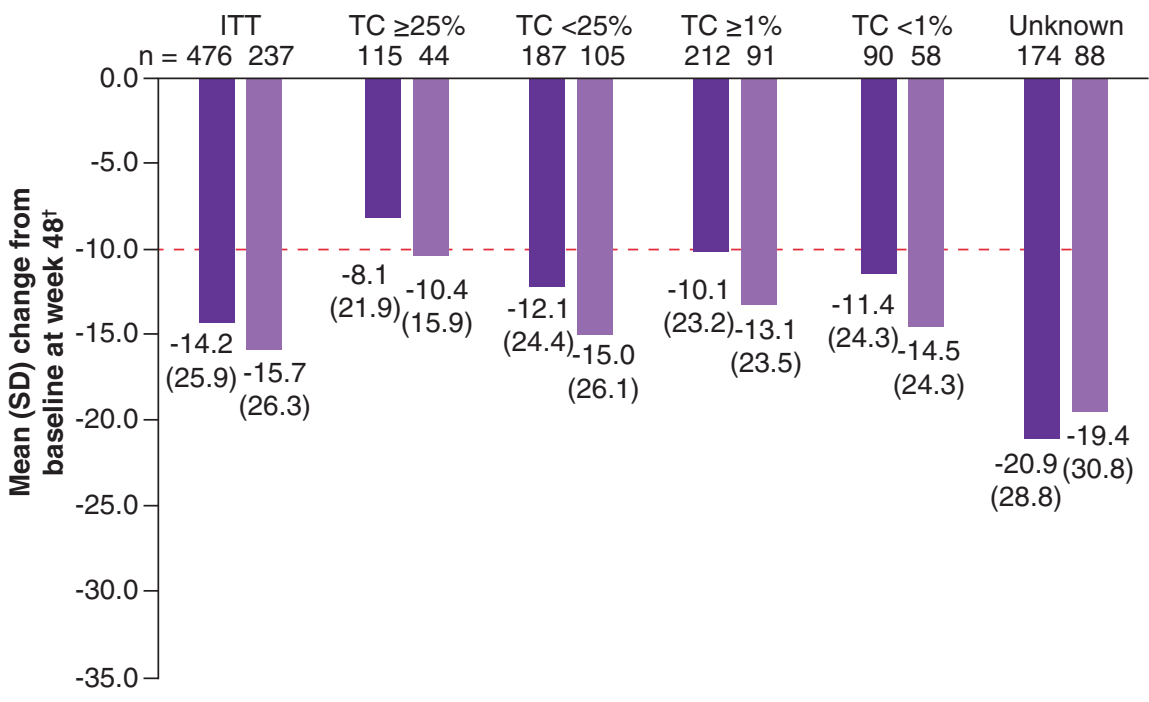

(B)

Alopecia (LC13)

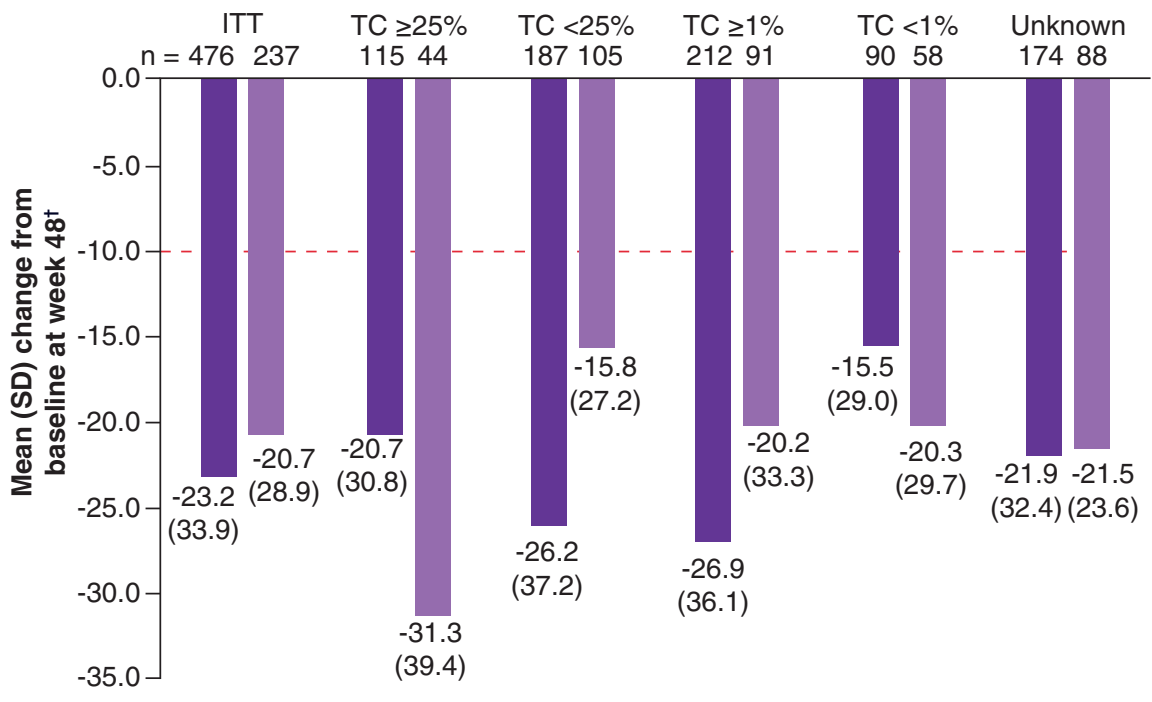

Durvalumab Placebo

Figure 2. Changes from baseline for dysphagia (A) and alopecia (B) in the intent-to-treat population and across all PD-L1 subgroups. Data gathered from the EORTC QLQ-C30 and EORTC QLQ-LC13 questionnaires. Dashed line indicates the threshold for clinically significant improvement from baseline; for symptoms, this was defined as a $\geq 10$-point decrease in score. C30 global health status/QoL and functional scales are based on patients with baseline scores $\geq 10$. C30 and LC13 symptom scales are based on patients with baseline scores $\leq 90$. Data cut-off: $22 \mathrm{March}$ 2018; median follow-up: 25.2 months (range: 0.2-43.1).

${ }^{\dagger}$ Mean difference between absolute baseline and week 48 scores, not the difference between the mean baseline and mean week 48 scores. A negative change from baseline is improvement for symptoms.

EORTC QLQ-C30: European Organisation for Research and Treatment of Cancer Quality of Life Questionnaire - Core 30; EORTC QLQ-LC13: European Organisation for Research and Treatment of Cancer Quality of Life Questionnaire Lung Cancer 13; ITT: Intent-to-treat; n: Number with improvements; N: Number of patients; QoL: Quality of life; SD: Standard deviation; TC: Tumor cell.

ITT data adapted from [13]. 
(A) PD-L1 TC $\geq 25 \%$

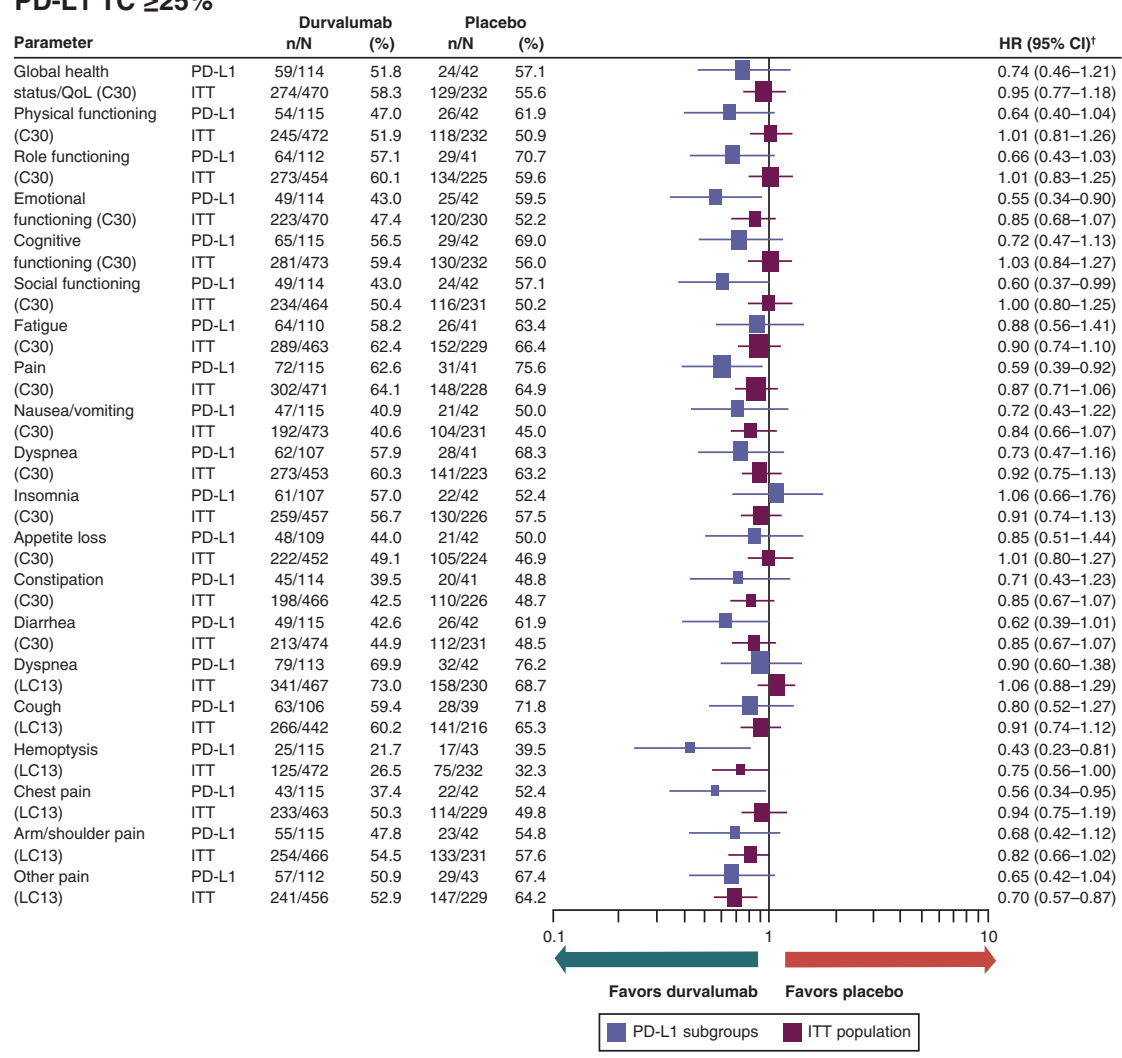

(B) PD-L1 TC $<25 \%$

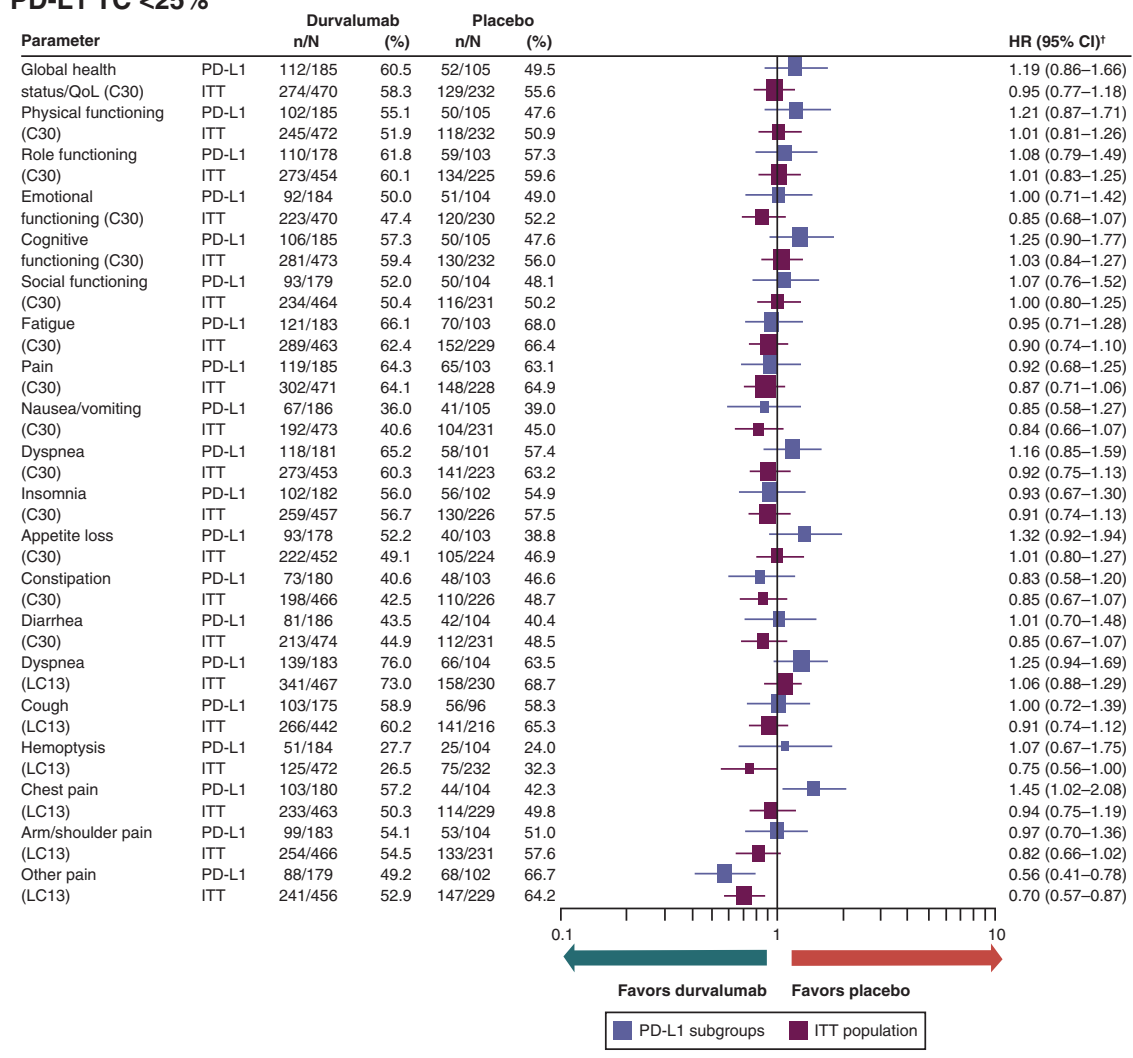

Figure 3. Time to deterioration of symptoms, functioning and global health status/quality of life in the intent-to-treat population and across PD-L1 subgroups defined by expression levels of (A) tumor cell $\geq 25 \%$, (B) tumor cell $<25 \%$, (C) unknown, (D) tumor cell $\geq 1 \%$ and $(E)$ tumor cell $<1 \%$. 
(C) PD-L1 Unknown

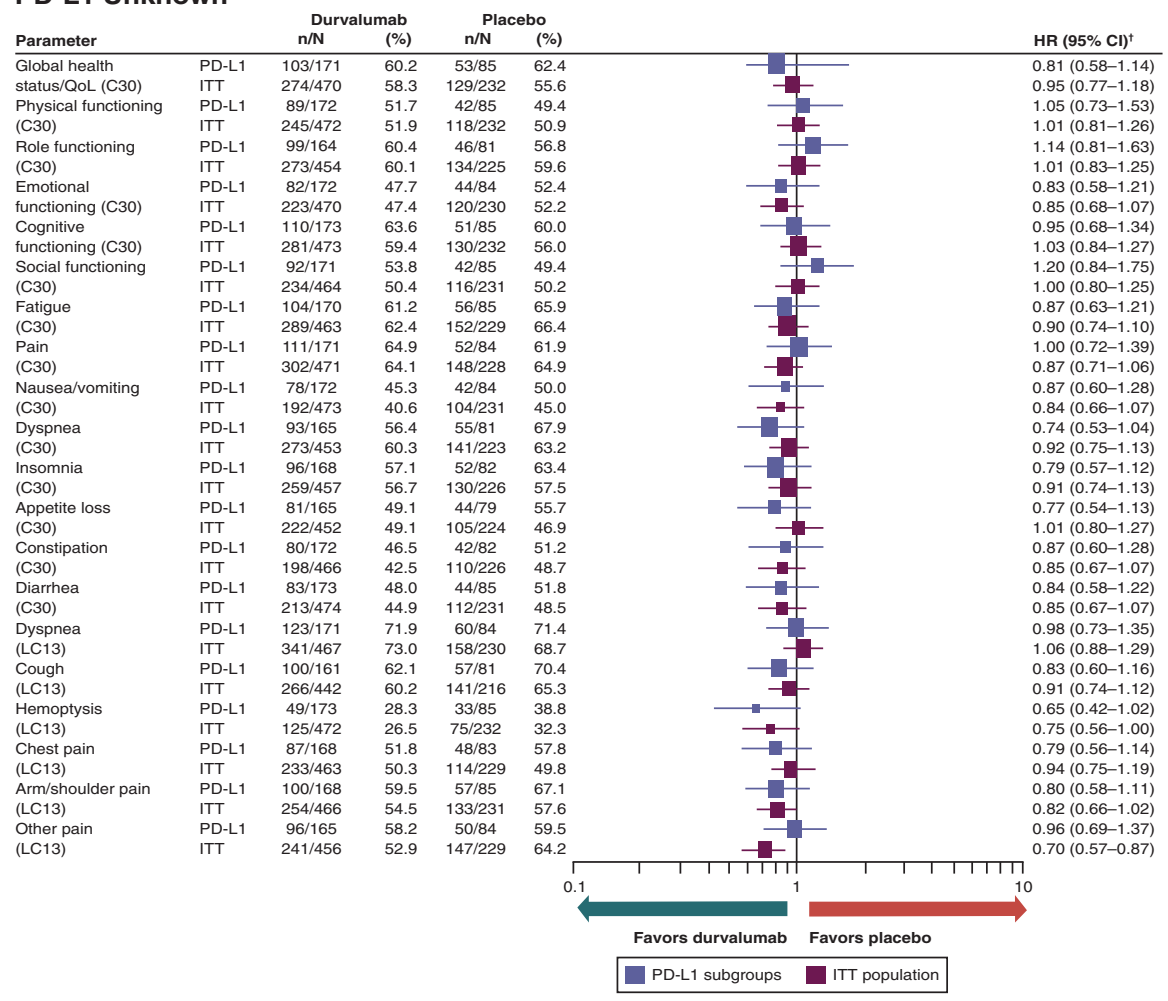

(D) PD-L1 TC $\geq 1 \%$

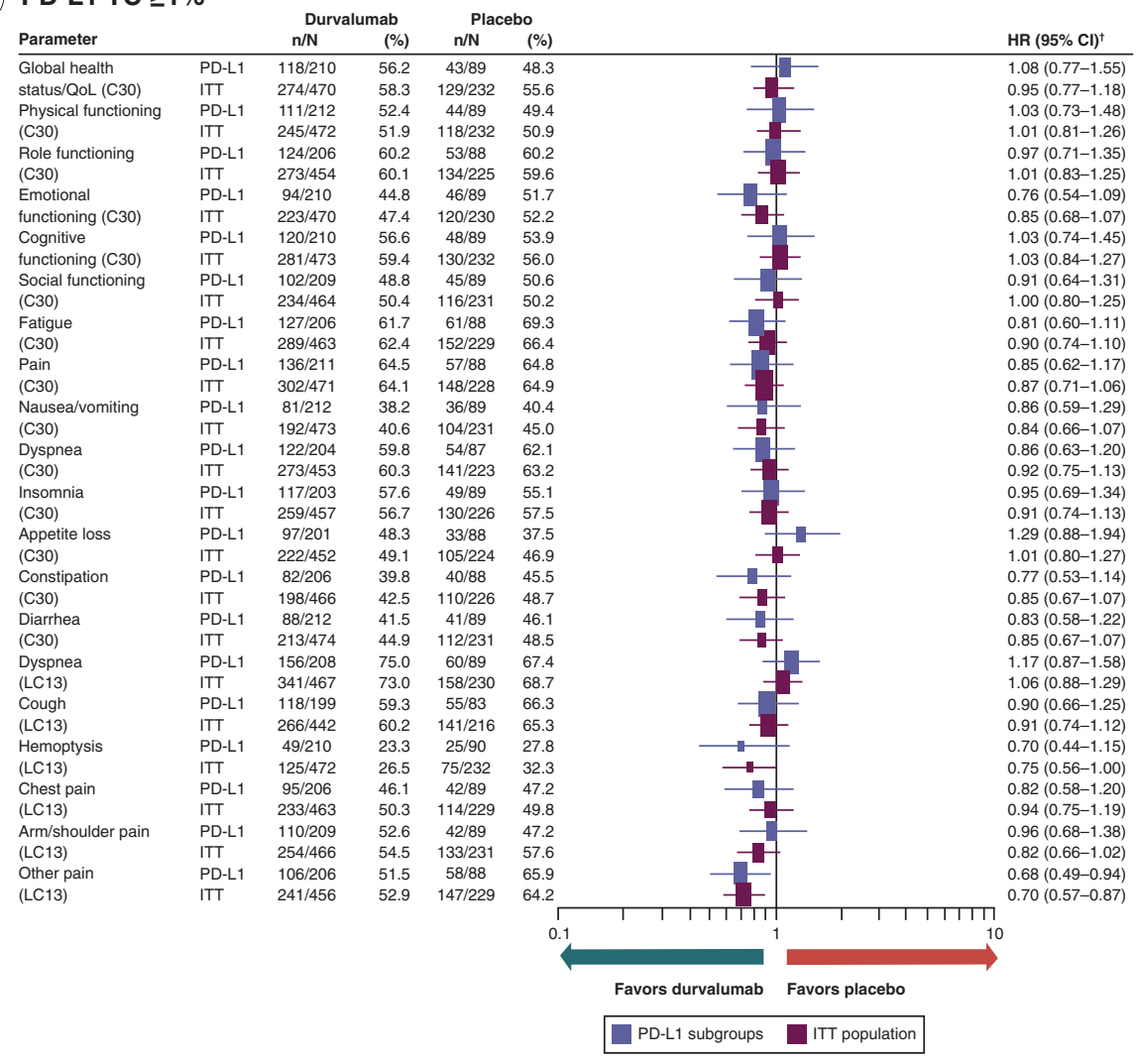

Figure 3. Time to deterioration of symptoms, functioning and global health status/quality of life in the intent-to-treat population and across PD-L1 subgroups defined by expression levels of (A) tumor cell $\geq 25 \%$, (B) tumor cell $<25 \%$, (C) unknown, (D) tumor cell $\geq 1 \%$ and $(E)$ tumor cell $<1 \%$ (cont.). 


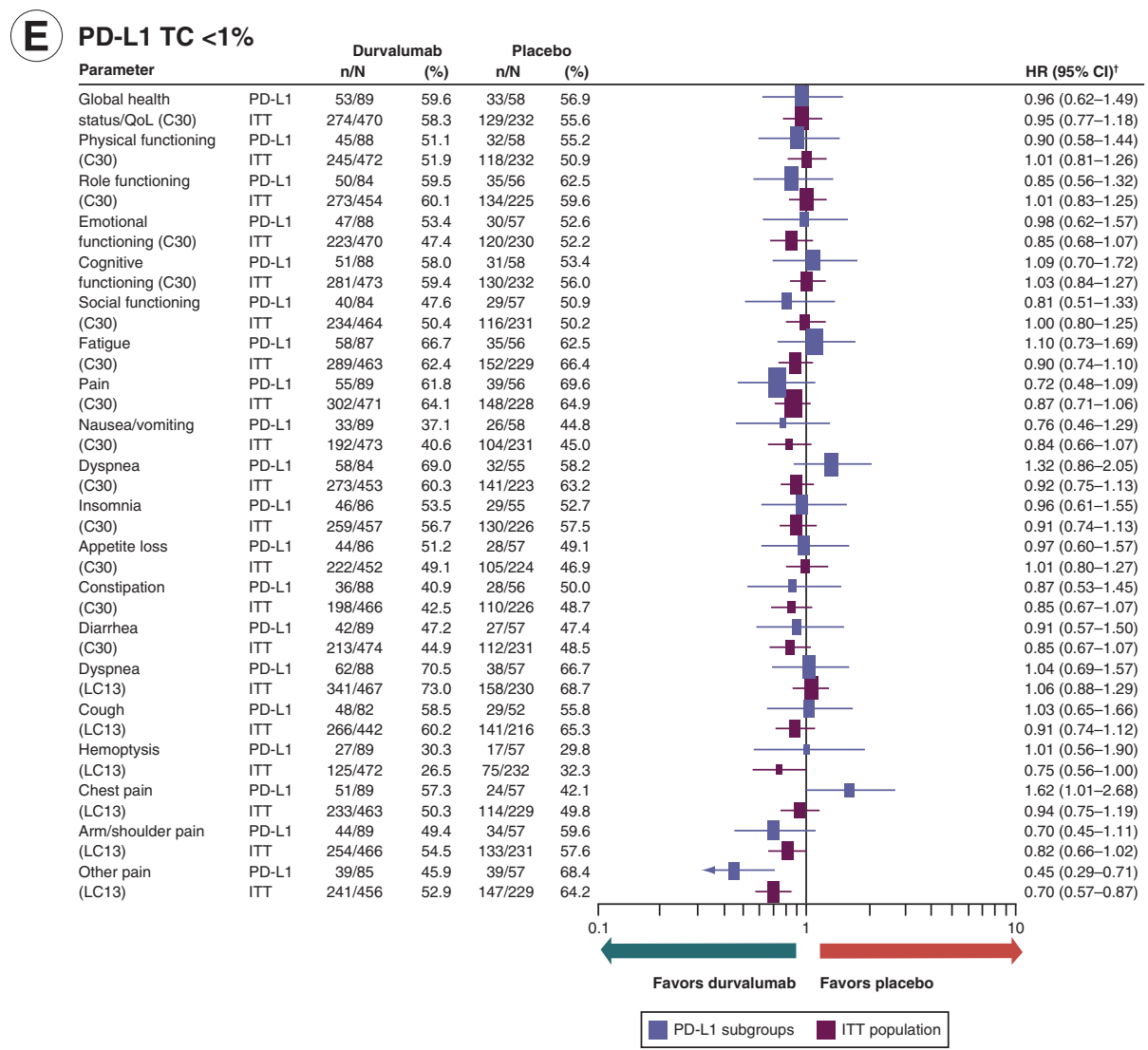

Figure 3. Time to deterioration of symptoms, functioning and global health status/quality of life in the intent-to-treat population and across PD-L1 subgroups defined by expression levels of (A) tumor cell $\geq 25 \%$, (B) tumor cell $<25 \%$, (C) unknown, (D) tumor cell $\geq 1 \%$ and $(E)$ tumor cell $<1 \%$ (cont.).

Data gathered from the EORTC QLQ-C30 (C30) and EORTC QLQ-LC13 (LC13). C30 global health status/QoL and functional scales are based on patients with baseline scores $\geq 10$. C30 and LC13 symptom scales are based on patients with baseline scores $\leq 90$. HR $<1$ favors durvalumab. Data cut-off: 22 March 2018; median follow-up: 25.2 months (range: $0.2-43.1$ ).

${ }^{\dagger} \mathrm{HRs}$ and corresponding $95 \% \mathrm{Cls}$ were calculated using Cox proportional-hazards models. The analysis was adjusted for the trial stratification factors for the ITT population, and an unstratified approach was used for analysis within PD-L1 subgroups. HRs and 95\% Cls were not calculated if the subgroup had fewer than 20 events.

EORTC QLQ-C30: European Organisation for Research and Treatment of Cancer Quality of Life Questionnaire - Core 30; EORTC QLQ-LC13: European Organisation for Research and Treatment of Cancer Quality of Life Questionnaire Lung Cancer 13; HR: Hazard ratio; ITT: Intent-to-treat; n: Number with improvements; N: Number of patients; QoL: Quality of life; TC: Tumor cell; TTD: Time to deterioration.

ITT data adapted from [13].

longer with durvalumab, versus placebo, among patients with PD-L1 TC $\geq 25 \%$. In addition, TTD of hemoptysis was longer with durvalumab compared with placebo among patients with PD-L1 TC $\geq 25 \%$. The findings of an additional analysis of TTD (where any clinically relevant deterioration observed at a given timepoint had to be confirmed at the next timepoint) were generally consistent with the main analysis (Supplementary Table 5); of note, with the exception of patients with PD-L1 TC $\geq 25 \%$, no clinically important between-arm differences in TTD of chest pain were observed for any of the PD-L1 subgroups in this additional analysis.

For the CRT subgroups, findings regarding TTD were also generally consistent with the ITT population. There were no clinically important between-arm differences in most of the key symptoms and global health status/QoL across most of the subgroups (Figure 4). However, TTD of hemoptysis was longer with durvalumab, versus placebo, among patients who received cisplatin, patients who did not receive taxane-based $\mathrm{CT}$, and patients randomized to study treatment $<14$ days after completing RT. Additionally, TTD of chest pain was longer with durvalumab, versus placebo, among patients who received a RT dose $<60 \mathrm{~Gy}$. 
Figure 4. Time to deterioration of key symptoms and global health status/quality of life across all chemoradiotherapy subgroups. Data gathered from the EORTC QLQ-C30 (C30) and EORTC QLQ-LC13 (LC13). C30 global health status/QoL and functional scales are based on patients with baseline scores $\geq 10$. C30 and LC13 symptom scales are based on patients with baseline scores $\leq 90$. HR $<1$ favors durvalumab.

${ }^{\dagger} \mathrm{HRs}$ and corresponding $95 \% \mathrm{Cls}$ were calculated using Cox proportional-hazards models. The analysis was adjusted for the trial stratification factors for the ITT population, and an unstratified approach was used for analysis within CRT subgroups. HRs and $95 \% \mathrm{Cls}$ were not calculated if the subgroup had fewer than 20 events.

Data cut-off: 22 March 2018; median follow-up: 25.2 months (range: 0.2-43.1).

CRT: Chemoradiotherapy; EORTC QLQ-C30: European Organisation for Research and Treatment of Cancer Quality of Life Questionnaire - Core 30; EORTC QLQ-LC13: European

Organisation for Research and Treatment of Cancer Quality of Life Questionnaire - Lung Cancer 13; Gy, units of gray; HR: Hazard ratio; ITT: Intent-to-treat; $\mathrm{n}$ : Number of events; N: Number of patients; QoL: Quality of life; RT: Radiotherapy; TTD: Time to deterioration.

ITT data adapted from [13].

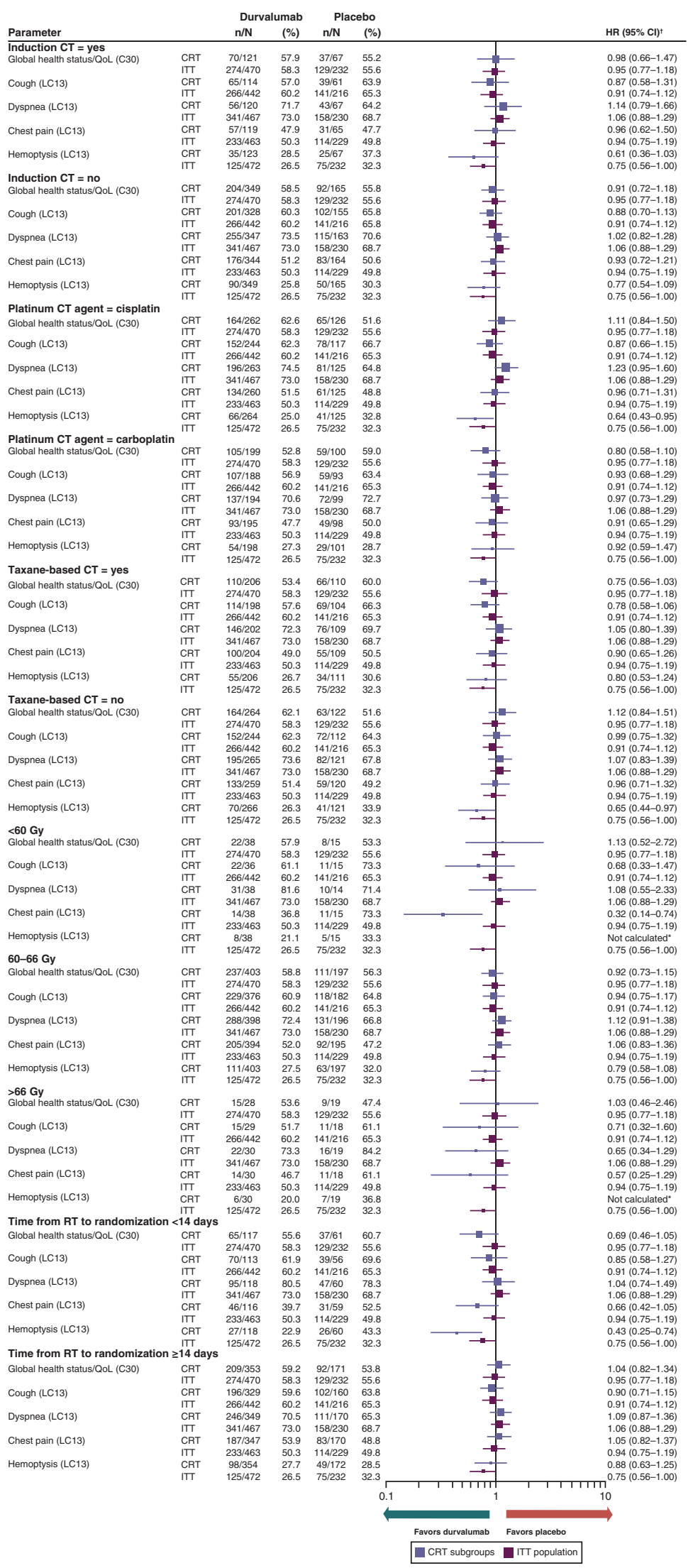




\section{Improvement rate}

Across the PD-L1 subgroups, results for the analysis of the proportion of patients with clinically relevant improvements in symptoms, functioning and global health status/QoL were generally consistent with findings in the ITT population, with treatment effects (ORs) and 95\% CIs overlapping between these populations for most scores (Figure 5). In general, there were no clinically important between-arm differences in improvement rate for the key symptoms of cough, dyspnea, chest pain, hemoptysis (all EORTC QLQ-LC13) and global health status/QoL (EORTC QLQ-C30). However, clinically important between-arm differences in improvement rate were observed for cough among patients with PD-L1 TC $<25 \%$ (favoring durvalumab) and for chest pain among patients with unknown PD-L1 status (favoring placebo).

For the CRT subgroups, findings regarding improvement rates were also generally consistent with the ITT population. There were no clinically important between-arm differences for the key symptoms and global health status/QoL across most of the subgroups (Figure 6). However, clinically important between-arm differences in improvement rate were observed for dyspnea among patients who received taxane-based CT and patients randomized to study treatment $<14$ days after completing RT (both favoring placebo). In addition, clinically important between-arm differences were observed for cough among patients who did not receive taxane-based CT (favoring durvalumab) and global health status/QoL among patients randomized to study treatment $<14$ days after completing RT (favoring placebo).

\section{Discussion}

The findings of this exploratory analysis indicate that treatment with durvalumab (compared with placebo) did not compromise symptoms, functioning or global health status/QoL, irrespective of tumoral PD-L1 expression (assessed in archived pre-CRT tumor tissue, where available) or variables related to prior CRT. Across the PD-L1 and CRT subgroups, scores for key PROs remained broadly stable in patients receiving up to 12 months of treatment with durvalumab or placebo, with few clinically meaningful differences observed between the treatment arms. Moreover, in general, durvalumab did not detrimentally affect TTD or the proportion of patients whose scores improved (from baseline), for key PROs across all analyzed subgroups, consistent with findings for the ITT population [13]. This represents, to the best of our knowledge, the first assessment of PROs according to these variables in a large Phase III trial within this disease setting. In addition, these findings further support the use of the PACIFIC regimen as the standard of care in stage III, unresectable NSCLC, irrespective of PD-L1 expression status or CRT-related treatment variables.

Despite demonstrated survival benefit with durvalumab versus placebo, improvements in PROs had not been expected due to the lower symptom burden and better baseline function of this population compared with patients in more advanced disease settings. In addition, the symptom burden at the time of progression was expected to be relatively low, given the frequency of surveillance imaging.

The findings of a previous exploratory analysis from PACIFIC indicated that clinical outcomes across the CRT subgroups were generally consistent with those observed in the ITT population [29]. When considered together with the limited differences in PROs across the CRT subgroups observed in the present study, the available data support the use of durvalumab in this setting irrespective of variables related to prior CRT.

Consistent with the ITT population, across all PD-L1 subgroups, clinically meaningful improvements were seen in alopecia and dysphagia irrespective of treatment arm (with the exception of dysphagia in patients with PD-L1 TC $\geq 25 \%$ who were randomized to receive durvalumab). As these symptoms were originally included in the EORTC QLQ-LC13 due to their association with CRT, and broadly similar changes were reported in both the durvalumab and placebo arms, the improvements recorded over the course of the present study were likely due to recovery from the side effects of CRT over time rather than any benefit derived from durvalumab [33].

The PROs data reported here provide additional context to the efficacy and safety data reported previously for PD-L1 subgroups from the PACIFIC trial [18]. Data from the EORTC QLQ-C30 and EORTC QLQ-LC30 questionnaires indicate that, irrespective of PD-L1 expression, durvalumab did not detrimentally affect symptoms, functioning or global health status/QoL. Alongside the manageable safety profile associated with durvalumab (reported in the ITT population and across all analyzed PD-L1 subgroups) [5,6,18], these PROs data have implications for potential future prospective investigations regarding clinical outcomes with the PACIFIC regimen, indicating that durvalumab can be administered safely and without compromising patient QoL irrespective of PD-L1 status. This is also important when considering the ethical validity of future studies of ICI in the post-CRT setting, which may enrol patients irrespective of tumor PD-L1 expression status. 


\section{(A) PD-L1 TC $\geq 25 \%$}

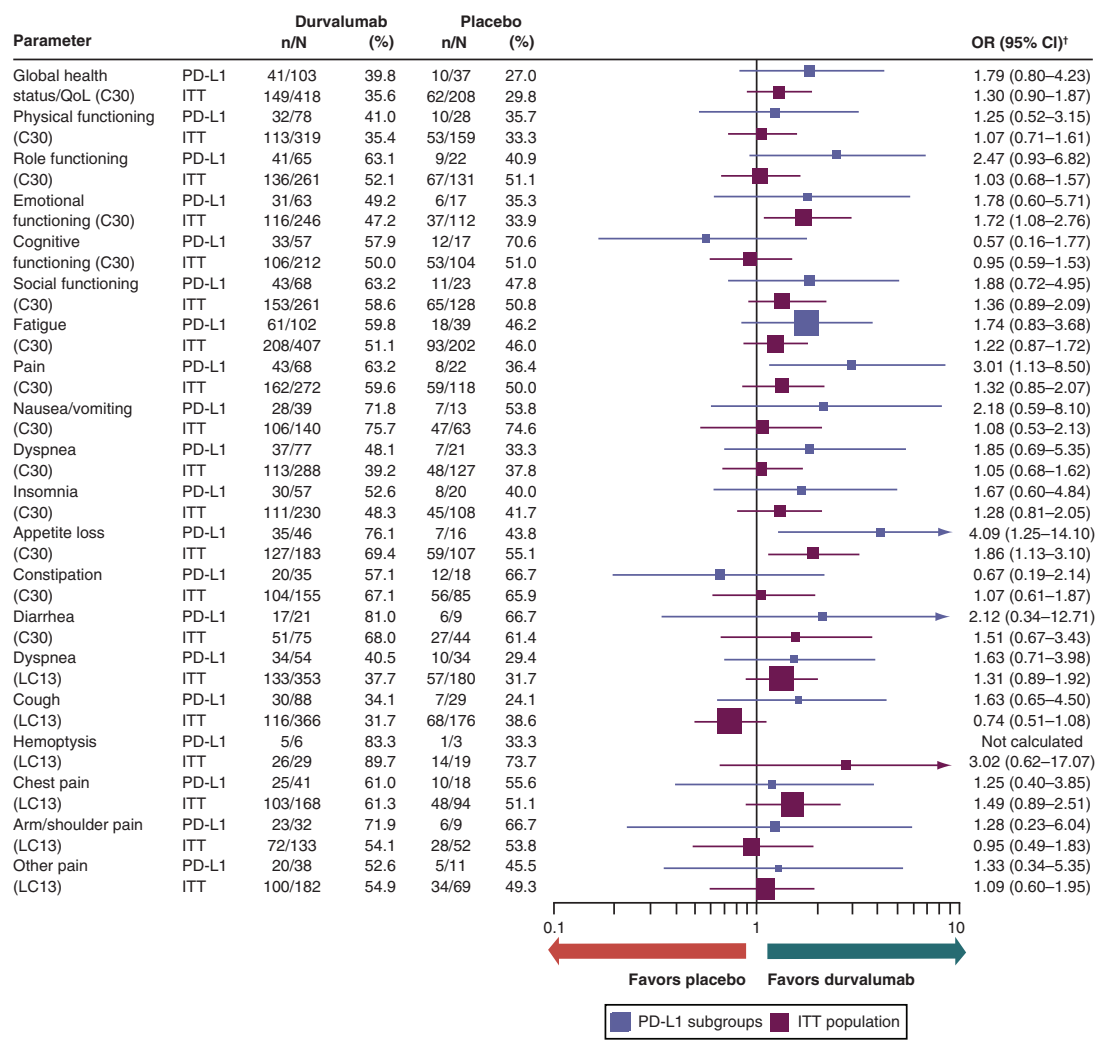

(B) PD-L1 TC $<25 \%$

\begin{tabular}{|c|c|c|c|c|c|c|c|c|}
\hline \multirow[b]{2}{*}{ Parameter } & & \multicolumn{2}{|c|}{ Durvalumab } & \multicolumn{2}{|c|}{ Placebo } & & & \multirow[b]{2}{*}{ OR $(95 \% \mathrm{Cl})^{\dagger}$} \\
\hline & & $n / N$ & $(\%)$ & $n / N$ & $(\%)$ & & & \\
\hline Global health & PD-L1 & $59 / 163$ & 36.2 & $36 / 97$ & 37.1 & & & $0.96(0.57-1.63)$ \\
\hline status/QoL (C30) & ITT & $149 / 418$ & 35.6 & $62 / 208$ & 29.8 & & & $1.30(0.90-1.87)$ \\
\hline Physical functioning & PD-L1 & $39 / 124$ & 31.5 & $27 / 71$ & 38.0 & & & $0.75(0.41-1.38)$ \\
\hline (C30) & ITT & $113 / 319$ & 35.4 & $53 / 159$ & 33.3 & & & $1.07(0.71-1.61)$ \\
\hline Role functioning & PD-L1 & $50 / 95$ & 52.6 & $34 / 58$ & 58.6 & & & $0.78(0.40-1.51)$ \\
\hline (C30) & ITT & $136 / 261$ & 52.1 & $67 / 131$ & 51.1 & & & $1.03(0.68-1.57)$ \\
\hline Emotional & PD-L1 & $44 / 89$ & 49.4 & $18 / 51$ & 35.3 & & & $1.79(0.89-5.71)$ \\
\hline functioning (C30) & ITT & $116 / 246$ & 47.2 & $38 / 112$ & 33.9 & & & $1.72(1.08-2.76)$ \\
\hline Cognitive & PD-L1 & $40 / 75$ & 53.3 & $28 / 54$ & 51.9 & & & $1.06(0.53-2.14)$ \\
\hline functioning (С30) & ITT & $106 / 212$ & 50.0 & $53 / 104$ & 51.0 & & & $0.95(0.59-1.53)$ \\
\hline Social functioning & PD-L1 & $61 / 98$ & 62.2 & $32 / 53$ & 60.4 & & & $1.08(0.54-2.14)$ \\
\hline (С30) & ITT & $153 / 261$ & 58.6 & $65 / 128$ & 50.8 & & & $1.36(0.89-2.09)$ \\
\hline Fatigue & PD-L1 & $73 / 153$ & 47.7 & $45 / 86$ & 52.3 & & & $0.83(0.49-1.41)$ \\
\hline (C30) & ITT & $208 / 407$ & 51.1 & $93 / 202$ & 46.0 & & & $1.22(0.87-1.72)$ \\
\hline Pain & PD-L1 & $62 / 101$ & 61.4 & $31 / 52$ & 59.6 & & & $1.08(0.54-2.13)$ \\
\hline (C30) & ITT & $162 / 272$ & 59.6 & $59 / 118$ & 50.0 & & & $1.32(0.85-2.07)$ \\
\hline Nausea/vomiting & PD-L1 & $33 / 45$ & 73.3 & $20 / 27$ & 74.1 & & & $0.96(0.31-2.81)$ \\
\hline (C30) & ITT & $106 / 140$ & 75.7 & $47 / 127$ & 74.6 & & & $1.08(0.53-2.13)$ \\
\hline Dyspnea & PD-L1 & $41 / 109$ & 37.6 & $22 / 51$ & 38.5 & & & $0.96(0.51-1.83)$ \\
\hline (C30) & ITT & $113 / 288$ & 39.2 & $48 / 127$ & 37.8 & & & $1.05(0.68-1.62)$ \\
\hline Insomnia & PD-L1 & $42 / 84$ & 50.0 & $22 / 51$ & 43.1 & & & $1.32(0.66-2.67)$ \\
\hline (С30) & ITT & $111 / 230$ & 48.3 & $45 / 108$ & 41.7 & & & $1.28(0.81-2.05)$ \\
\hline Appetite loss & PD-L1 & $45 / 66$ & 68.2 & $31 / 48$ & 64.6 & & & $1.18(0.53-2.58)$ \\
\hline (C30) & ITT & $127 / 183$ & 69.4 & $59 / 107$ & 55.1 & & 둘 & $1.86(1.13-3.10)$ \\
\hline Constipation & PD-L1 & $44 / 57$ & 77.2 & $26 / 39$ & 66.7 & & & $0.69(0.68-4.24)$ \\
\hline (C30) & ITT & $104 / 155$ & 67.1 & $56 / 85$ & 65.9 & & & $1.07(0.61-1.87)$ \\
\hline Diarrhea & PD-L1 & $20 / 27$ & 74.1 & $13 / 24$ & 54.2 & & & $2.42(0.76-8.16)$ \\
\hline (C30) & ITT & $51 / 75$ & 68.0 & $27 / 44$ & 61.4 & & & $1.51(0.67-3.43)$ \\
\hline Dyspnea & PD-L1 & $54 / 137$ & 39.4 & $32 / 83$ & 38.6 & & & $1.04(0.59-1.82)$ \\
\hline (LC13) & ITT & $133 / 353$ & 37.7 & $57 / 180$ & 31.7 & & & $1.31(0.89-1.92)$ \\
\hline Cough & PD-L1 & $47 / 146$ & 32.2 & $39 / 80$ & 47.6 & & & $1.52(0.30-0.91)$ \\
\hline (LC13) & ITT & $116 / 366$ & 31.7 & $68 / 173$ & 38.6 & & & $0.74(0.51-1.08)$ \\
\hline Hemoptysis & PD-L1 & $12 / 12$ & 100.0 & $10 / 13$ & 76.9 & & & Not calculated \\
\hline (LC13) & ITT & $26 / 29$ & 89.7 & $14 / 19$ & 73.7 & & & $3.02(0.62-17.07)$ \\
\hline Chest pain & PD-L1 & $36 / 61$ & 59.0 & $25 / 42$ & 59.5 & & & $0.98(0.44-2.18)$ \\
\hline (LC13) & ITT & $103 / 168$ & 61.3 & $48 / 94$ & 51.1 & & & $1.49(0.89-2.51)$ \\
\hline Arm/shoulder pain & PD-L1 & $25 / 48$ & 52.1 & $10 / 19$ & 52.6 & & & $0.98(0.33-2.85)$ \\
\hline (LC13) & ITT & $72 / 133$ & 54.1 & $28 / 52$ & 53.8 & & & $0.95(0.49-1.83)$ \\
\hline \multirow{5}{*}{$\begin{array}{l}\text { Other pain } \\
\text { (LC13) }\end{array}$} & PD-L1 & $46 / 70$ & 65.7 & $15 / 32$ & 46.9 & & & $2.17(0.93-5.15)$ \\
\hline & ITT & $100 / 182$ & 54.9 & $34 / 69$ & 49.3 & & & $1.09(0.60-1.95)$ \\
\hline & & & & & & $\begin{array}{l}\square \\
0.1\end{array}$ & $\begin{array}{r}111010 \\
10\end{array}$ & \\
\hline & & & & & & Favors placebo & Favors durvalumab & \\
\hline & & & & & & PD-L1 subgroups & ITT population & \\
\hline
\end{tabular}

Figure 5. Improvement rates in symptoms, functioning and global health status/quality of life in the intent-to-treat population and across PD-L1 subgroups defined by expression levels of (A) tumor cell $\geq 25 \%$, (B) tumor cell $<25 \%$, (C) unknown, (D) tumor cell $\geq 1 \%$ and $(E)$ tumor cell $<1 \%$. 
(C) PD-L1 Unknown

\begin{tabular}{|c|c|c|c|c|c|}
\hline \multirow{2}{*}{ Parameter } & & \multicolumn{2}{|c|}{ urvalur } & \multicolumn{2}{|c|}{ Placebo } \\
\hline & & $n / N$ & (\%) & $n / N$ & \\
\hline Global health & PD-L1 & $49 / 152$ & 32.2 & $16 / 74$ & \\
\hline status/QoL (C30) & ITT & $149 / 418$ & 35.6 & $62 / 208$ & \\
\hline Physical functioning & PD-L1 & $42 / 117$ & 35.9 & $16 / 60$ & \\
\hline (С30) & ITT & $113 / 319$ & 35.4 & $53 / 159$ & \\
\hline Role functioning & PD-L1 & $45 / 101$ & 44.6 & $24 / 51$ & \\
\hline 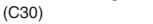 & ITT & $136 / 261$ & 52.1 & $67 / 131$ & \\
\hline Emotional & PD-L1 & $41 / 94$ & 43.6 & $14 / 44$ & \\
\hline functioning (С30) & ITT & $116 / 246$ & 47.2 & $38 / 112$ & \\
\hline Cognitive & PD-L1 & $33 / 80$ & 41.3 & $13 / 33$ & \\
\hline functioning (C30) & ITT & $106 / 212$ & 50.0 & $53 / 104$ & \\
\hline Social functioning & PD-L1 & $49 / 95$ & 51.6 & $22 / 52$ & \\
\hline (C30) & ITT & $153 / 261$ & 58.6 & $65 / 128$ & \\
\hline Fatigue & PD-L1 & $74 / 152$ & 48.7 & $30 / 77$ & \\
\hline (C30) & ITT & $208 / 407$ & 51.1 & $93 / 202$ & \\
\hline Pain & PD-L1 & $57 / 103$ & 55.3 & $20 / 44$ & \\
\hline (С30) & ITT & $162 / 272$ & 59.6 & $59 / 118$ & \\
\hline Nausea/vomiting & PD-L1 & $45 / 56$ & 80.4 & $20 / 23$ & \\
\hline (C30) & ITT & $106 / 140$ & 75.7 & $47 / 63$ & \\
\hline Dyspnea & PD-L1 & $35 / 102$ & 34.3 & $16 / 41$ & \\
\hline (С 30$)$ & ITT & $113 / 288$ & 39.2 & $48 / 127$ & \\
\hline Insomnia & PD-L1 & $39 / 89$ & 43.8 & $15 / 37$ & \\
\hline (C30) & ITT & $111 / 230$ & 48.3 & $45 / 108$ & \\
\hline Appetite loss & PD-L1 & $47 / 71$ & 66.2 & $21 / 43$ & \\
\hline (C30) & ITT & $127 / 183$ & 69.4 & $59 / 107$ & \\
\hline Constipation & PD-L1 & $40 / 63$ & 63.5 & $18 / 28$ & \\
\hline (C30) & ITT & $104 / 155$ & 67.1 & $56 / 85$ & \\
\hline Diarrhea & PD-L1 & $14 / 27$ & 51.9 & $8 / 11$ & \\
\hline (C30) & ITT & $51 / 75$ & 68.0 & $27 / 44$ & \\
\hline Dyspnea & PD-L1 & $45 / 132$ & 34.1 & $15 / 63$ & \\
\hline (LC13) & ITT & $133 / 353$ & 37.7 & $57 / 180$ & \\
\hline Cough & PD-L1 & $39 / 132$ & 29.5 & $22 / 65$ & \\
\hline (LC13) & ITT & $116 / 366$ & 31.7 & $68 / 176$ & \\
\hline Hemoptysis & PD-L1 & $9 / 11$ & 81.8 & $3 / 3$ & \\
\hline (LC13) & ITT & $26 / 29$ & 89.7 & $14 / 19$ & \\
\hline Chest pain & PD-L1 & $42 / 66$ & 63.6 & $13 / 34$ & \\
\hline (LC13) & ITT & $103 / 168$ & 61.3 & $48 / 94$ & \\
\hline Arm/shoulder pain & PD-L1 & $24 / 53$ & 45.3 & $12 / 24$ & \\
\hline (LC13) & ITT & $72 / 133$ & 54.1 & $28 / 52$ & \\
\hline Other pain & PD-L1 & $34 / 74$ & 45.9 & $14 / 26$ & \\
\hline (LC13) & & $100 / 182$ & 54.9 & $34 / 69$ & \\
\hline
\end{tabular}

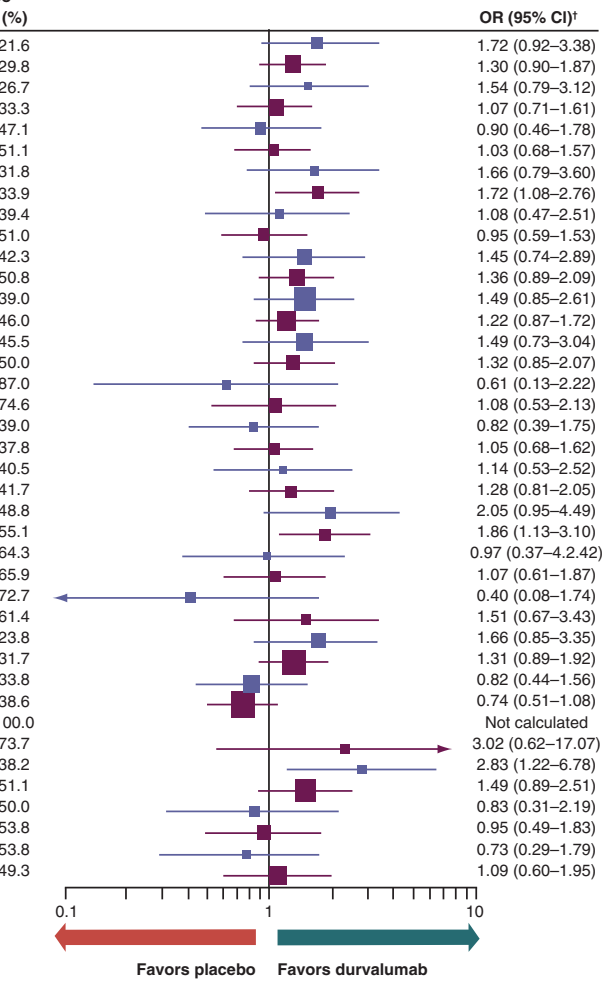

(D) PD-L1 TC $\geq 1 \%$

PD-L1 subgroups $\square$ ITT population

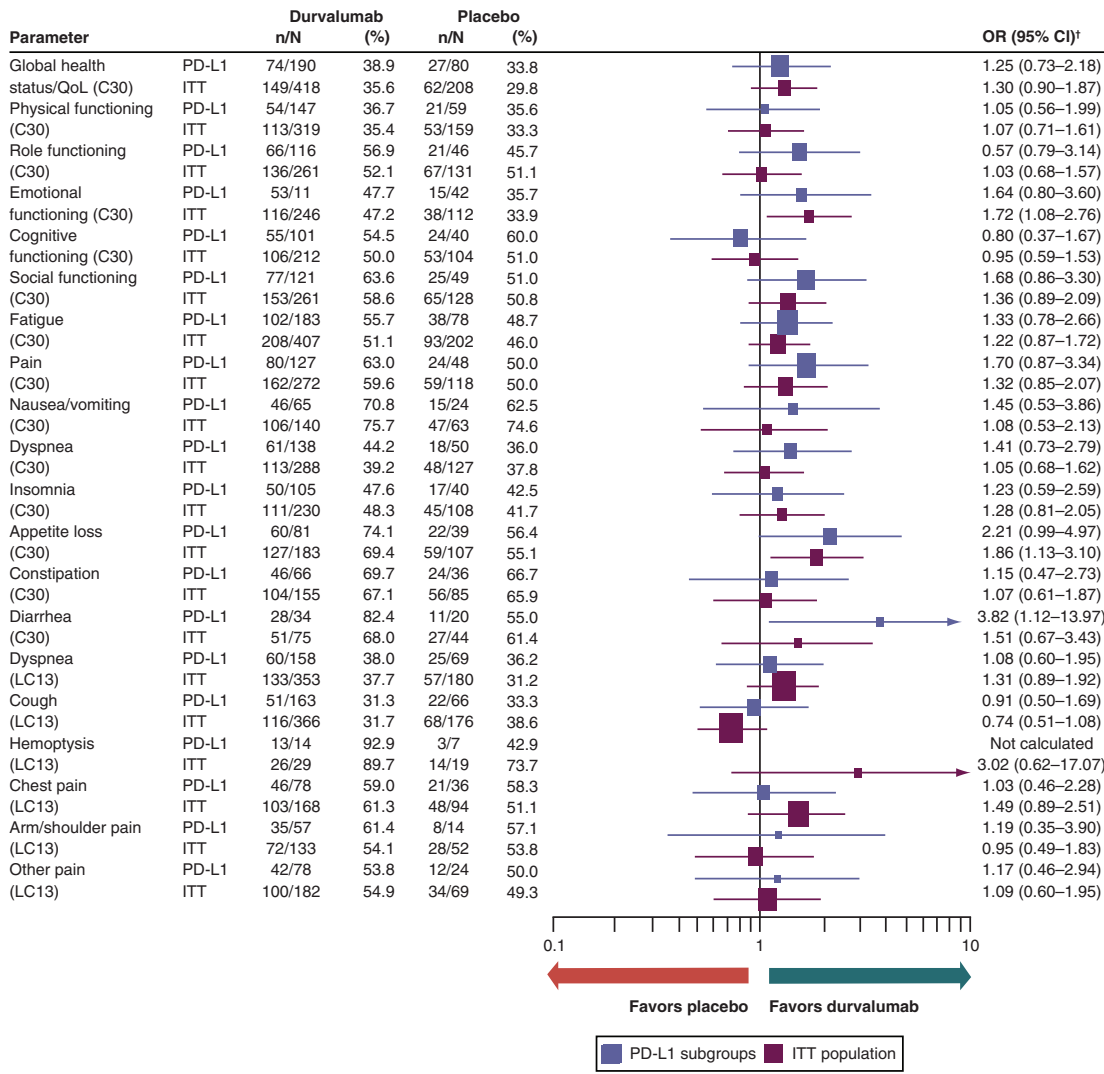

Figure 5. Improvement rates in symptoms, functioning and global health status/quality of life in the intent-to-treat population and across PD-L1 subgroups defined by expression levels of (A) tumor cell $\geq 25 \%$, (B) tumor cell $<25 \%$, (C) unknown, (D) tumor cell $\geq 1 \%$ and $(E)$ tumor cell $<1 \%$ (cont.). 


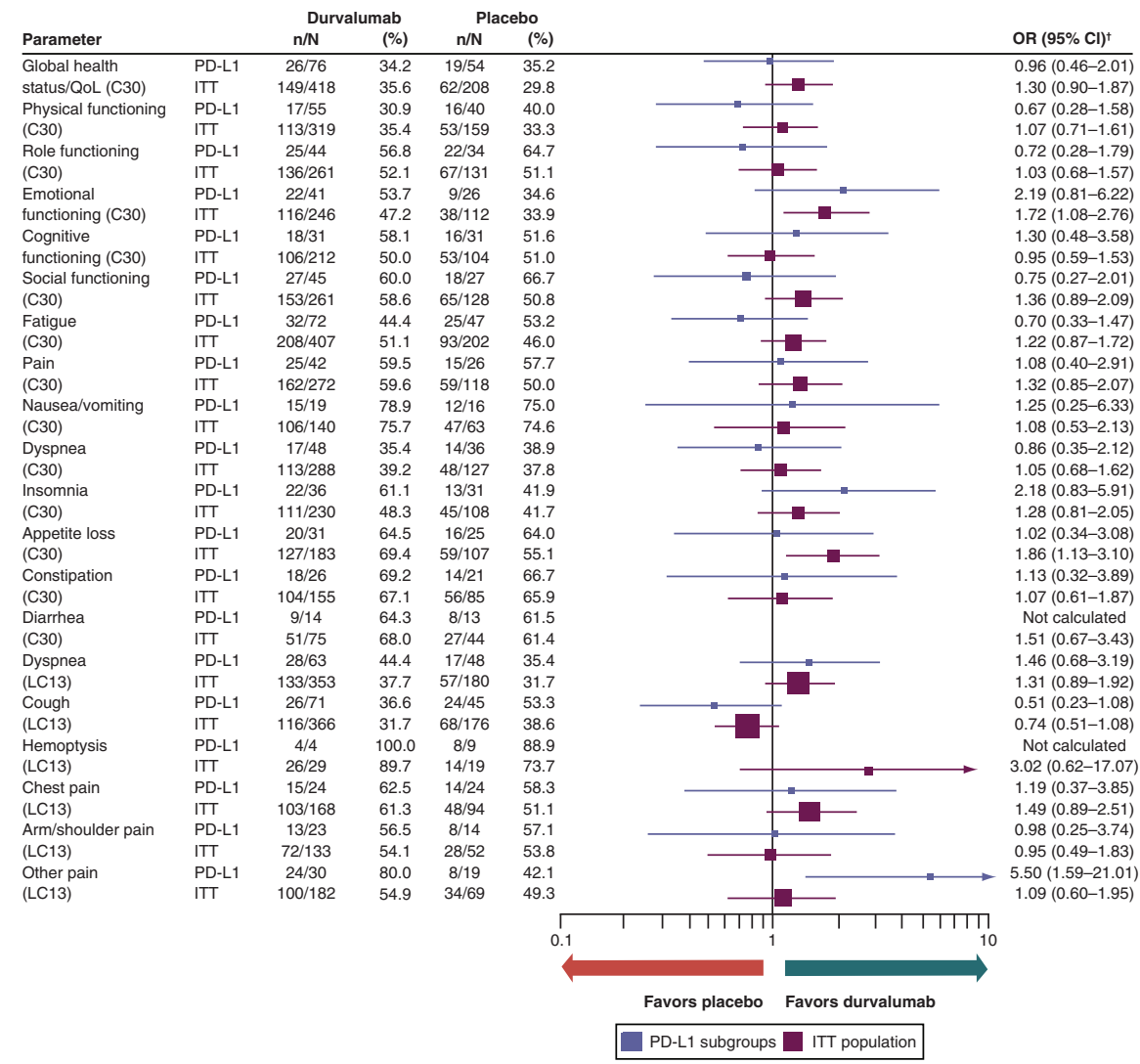

Figure 5. Improvement rates in symptoms, functioning and global health status/quality of life in the intent-to-treat population and across PD-L1 subgroups defined by expression levels of (A) tumor cell $\geq 25 \%$, (B) tumor cell $<25 \%$, (C) unknown, (D) tumor cell $\geq 1 \%$ and $(E)$ tumor cell $<1 \%$ (cont.).

Data gathered from the EORTC QLQ-C30 (C30) and EORTC QLQ-LC13 (LC13). C30 global health status/QoL and functional scales are based on patients with baseline scores $\geq 10$. C30 and LC13 symptom scales are based on patients with baseline scores $\leq 90$. OR $>1$ favors durvalumab.

†ORs and corresponding $95 \% \mathrm{Cls}$ were calculated using logistic regression. The analysis was adjusted for the trial stratification factors for the ITT population, and an unstratified approach was used for analysis within PD-L1 subgroups. ORs and $95 \% \mathrm{Cls}$ were not calculated if the subgroup had fewer than 20 events.

Data cut-off: 22 March 2018; median follow-up: 25.2 months (range: 0.2-43.1).

EORTC QLQ-C30: European Organisation for Research and Treatment of Cancer Quality of Life Questionnaire - Core 30; EORTC QLQ-LC13: European Organisation for Research and Treatment of Cancer Quality of Life Questionnaire Lung Cancer 13; ITT: Intent-to-treat; n: Number with improvements; N: Number of patients; OR: Odds ratio; QoL: Quality of life; TC: Tumor cell.

ITT data adapted from [13].

The relative importance of PD-L1 as a predictive biomarker for the efficacy of PD-1/PD-L1 ICI (particularly as pertains to specific individual ICIs) remains unclear [14]. No studies had definitively addressed this issue at the conception of the PACIFIC trial and so enrolment was not restricted or stratified by PD-L1 expression; the study was instead designed to assess the primary end points of PFS and OS in the ITT population $[5,6,34]$. The provision of a tumor tissue sample for evaluation of PD-L1 status was optional; PD-L1 status was only known for 63\% $(\mathrm{n}=451)$ of randomized patients and an analysis of efficacy by PD-L1 expression (using a prespecified TC threshold of $25 \%$ ) was initially included as a prespecified exploratory end point. However, an additional exploratory analysis using a post-hoc PD-L1 TC threshold of $1 \%$ was also undertaken at the request of the EMA. Previously described limitations in the PACIFIC trial design meant the detection of differences in efficacy within the relatively small subgroups defined by these PD-L1 TC thresholds was not possible; while PFS benefit with durvalumab (versus placebo) was observed across all PD-L1 subgroups, OS benefit with durvalumab was not demonstrated among patients with PD-L1 TC $<1 \%$ [6,7], possibly due to overperformance of the placebo arm in this subgroup, as 


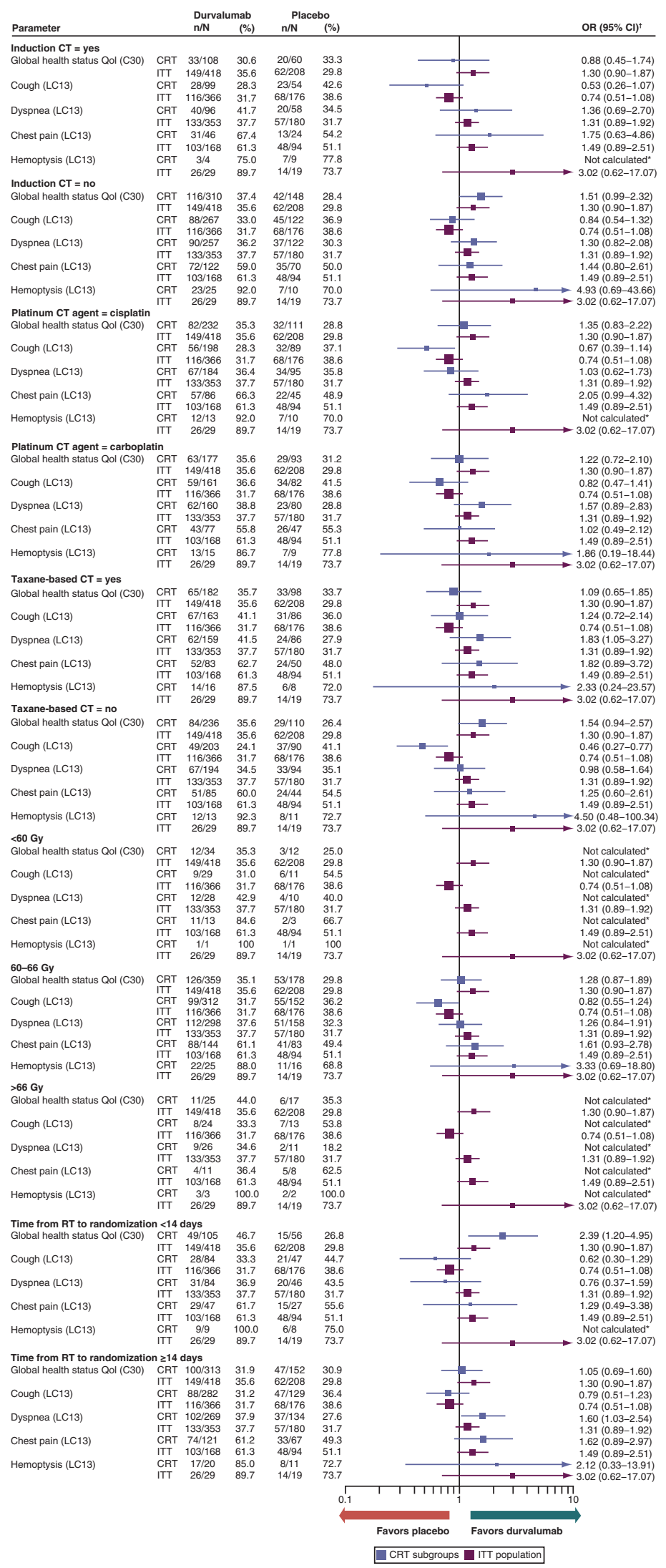

Figure 6. Improvement rates in key symptoms and global health status/quality of life across all chemoradiotherapy subgroups. Data gathered from the EORTC QLQ-C30 (C30) and EORTC QLQ-LC13 (LC13). C30 global health status/QoL and functional scales are based on patients with baseline scores $\geq 10$. C30 and LC13 symptom scales are based on patients with baseline scores $\leq 90$. OR $>1$ favors durvalumab. $\dagger$ ORs and corresponding $95 \% \mathrm{Cls}$ were calculated using logistic regression. The analysis was adjusted for the trial stratification factors for the ITT population, and an unstratified approach was used for analysis within CRT subgroups. ORs and 95\% Cls were not calculated if the subgroup had fewer than 20 events. Data cut-off: 22 March 2018; median follow-up: 25.2 months (range: 0.2-43.1). CRT: Chemoradiotherapy; EORTC QLQ-C30: European Organisation for Research and Treatment of Cancer Quality of Life Questionnaire - Core 30; EORTC QLQ-LC13: European Organisation for Research and Treatment of Cancer Quality of Life Questionnaire - Lung Cancer 13; Gy: Units of gray; $\mathrm{n}$ : Number with improvements; $\mathrm{N}$ : Number of patients; OR: Odds ratio; QoL: Quality of life; RT: Radiotherapy. ITT data adapted from [13]. 
indicated by previous analyses [18]. Therefore, further evaluation of PROs within these parameters was warranted to provide a more comprehensive understanding regarding the benefit/risk profile of durvalumab across PD-L1 patient populations. Cumulatively, the outcomes with durvalumab among patients with PD-L1 TC $<1 \%$ show a broadly favourable risk/benefit ratio, as evidenced by the improved PFS in this subgroup (albeit without a corresponding OS benefit), along with a manageable safety profile and no apparent detriment to PROs [18].

The limitations of the analyses of PROs in PD-L1 subgroups mirror those of the previously reported analyses of efficacy and safety outcomes from the PACIFIC trial according to PD-L1 status and were primarily a function of their retrospective nature $[18,34]$. Key limitations included the incomplete provision of tumor tissue samples within the ITT population (i.e., 37\% [ $\mathrm{n}=262]$ of patients had unknown PD-L1 status) and the consequent relatively small size of each of the PD-L1 subgroups. This was especially evident in the PD-L1 TC $<1 \%$ subgroup, which represented approximately $21 \%(n=148)$ of the ITT population across both treatment arms. As trial enrolment was not stratified according to PD-L1 status, these analyses are limited by possible imbalances in potentially prognostic baseline characteristics both between the subgroups, and between treatment arms within the subgroups; indeed, a higher proportion of patients within the placebo arm of the TC $<1 \%$ subgroup (relative to the durvalumab arm) were aged $<65$ years, white and female, and had non-squamous histology and stage IIIA disease [18]. Moreover, among the $37 \%$ of patients with unknown PD-L1 status, there is no guarantee that tumor samples were missing at random and that patients of varying PD-L1 expression levels were distributed in a balanced way across the treatment arms, representing a potential source of bias [18]. An additional limitation was the timing of tumor tissue sample collection; as samples were archival, and therefore collected pre-CRT, the IHC assay results do not reflect possible changes in PD-L1 expression that may have occurred as a result of CRT (previous data indicate that RT and CT may lead to upregulation of PD-L1 expression on tumor cells) $[21,27,28,35,36]$.

As noted for the PD-L1 subgroups, the analyses of PROs in CRT subgroups were also limited by the lack of stratification of trial enrollment according to CRT-related variables and the relatively small sizes of certain subgroups (in particular, the RT dose $<60 \mathrm{~Gy}[\mathrm{n}=53]$ and $>66 \mathrm{~Gy}[\mathrm{n}=49]$ subgroups).

\section{Conclusion}

In the PACIFIC trial, PROs for all PD-L1 subgroups, including patients with PD-L1 TC $<1 \%$, were generally consistent with findings in the ITT population, suggesting that durvalumab (compared with placebo) had no detrimental impact on patients' symptoms, functioning, or global health status/QoL regardless of PD-L1 status. Key PRO end points were also generally consistent with the ITT population across subgroups defined by variables related to prior CRT. As statistically significant and clinically meaningful improvements in PFS and OS were observed with durvalumab (versus placebo) in the ITT population, alongside a manageable safety profile [5,6], the consistency of the observed PRO data irrespective of PD-L1 status and CRT-related variables, further supports the position of PACIFIC regimen as standard of care in stage III, unresectable NSCLC who have no evidence of disease progression after definitive CRT.

\section{Supplementary data}

To view the supplementary data that accompany this paper please visit the journal website at: www.futuremedicine.com/doi/sup $\mathrm{pl} / 10.2217 /$ fon-2020-1102

\section{Acknowledgments}

The authors would like to thank the patients, their families and caregivers, and all investigators involved in this study.

\section{Financial \& competing interests disclosure}

This study (ClinicalTrials.gov: NCT02125461) was funded by AstraZeneca. C Faivre-Finn is supported by a grant from the NIHR Manchester Biomedical Research Centre. MC Garassino has received advisory fees from AstraZeneca, Bristol-Myers Squibb, Clovis Oncology, Genentech/Roche, Eli Lilly, Trovagene, Vortex Biosciences, Amgen and Celgene; speaker's bureau fees from Merck; and research funding from Eli Lilly, Genentech/Roche, Astex Pharmaceuticals, Clovis Oncology, Bristol-Myers Squibb, AstraZeneca, Threshold Pharmaceuticals, Array BioPharma, Celgene, AbbVie, Astellas Pharma, Corvus Pharmaceuticals and Spectrum Pharmaceuticals. L Paz-Ares has received advisory fees from MSD, Bristol-Myers Squibb, Roche, AstraZeneca, Eli Lilly, Merck, Novartis, Amgen, Incyte, Takeda, Blueprint, Bayer, PharmaMar and Ipsen; speaker's bureau fees from MSD, Bristol-Myers Squibb, Roche, AstraZeneca and Eli Lilly; travel, accommodations and expenses from MSD, Roche and AstraZeneca; and is an editorial board member for Altum Sequencing and Genomica. R Hui has received advisory fees from AstraZeneca, Bristol-Myers Squibb, Eli Lilly, MSD, Novar- 
tis and Roche; and speaker's bureau fees from MSD, Novartis and Roche. C Faivre-Finn reports research funding from AstraZeneca and Elekta; and travel, accommodations and expenses from AstraZeneca and Elekta. A Spira has received advisory fees from Array BioPharma and Incyte; honoraria from CytomX Therapeutics, AstraZeneca and Merck; research funding from LAM Therapeutics; and institutional research support from Roche, AstraZeneca, Boehringer Ingelheim, Astellas Pharma, Novartis, NewLink Genetics, Incyte, AbbVie, Ignyta, LAM Therapeutics, TrovaGene, Takeda, MacroGenics, CytomX Therapeutics, Astex Pharmaceuticals, Bristol-Myers Squibb, Loxo and Arch Therapeutics. D Planchard has received advisory fees from AstraZeneca, Bristol-Myers Squibb, Boehringer Ingelheim, Celgene, Daiichi Sankyo, Eli Lilly, Merck, Novartis, Pfizer, prIME Oncology, Peer CME, Roche and Samsung; and reports clinical trials research as principal or co-investigator for AstraZeneca, Bristol-Myers Squibb, Boehringer Ingelheim, Eli Lilly, Merck, Novartis, Pfizer, Roche, Sanofi Aventis, Taiho Pharma, Novocure and Daiichi Sankyo. D Daniel has received institutional research support from AstraZeneca, Boehringer Ingelheim, Genentech/Roche, Eli Lilly, Celgene, Pfizer and G1 Therapeutics. D Vicente has received honoraria from AstraZeneca, Pfizer, Bristol-Myers Squibb, MSD and Roche; and research funding from AstraZeneca, Pfizer, MSD and Roche. C Langer has received advisory fees from AstraZeneca, Amgen, Merck, Genentech/Roche, Hospira, Gilead and Eli Lilly; and institutional research support from Merck, Eli Lilly, Inovio and Trizell. S Senan has received honoraria from AstraZeneca, Celgene, MSD and Varian Medical Systems; and research support from AstraZeneca, ViewRay Inc. and Varian Medical Systems. D Spigel has received consulting fees his to institution from Aptitude Health, Bayer, Bristol-Myers Squibb, Dracen Pharmaceuticals, EMD Serono, Exelixis, Genentech/Roche, GlaxoSmithKline, Iksuda Therapeutics, Intellisphere, Ipsen, Jazz Pharmaceuticals, Mirati Therapeutics, Molecular Templates, Novartis, Puma Biotechnology and Takeda; and institutional research support from AstraZeneca, Aeglea Biotherapeutics, Agios, Astellas Pharma, BIND Therapeutics, Bristol-Myers Squibb, Celgene, Celldex Therapeutics, Clovis, Daiichi Sankyo, Eisai, Eli Lilly, EMD Serono, Genentech/Roche, G1 Therapeutics, GlaxoSmithKline, GRAIL, ImClone Systems, Ipsen, Janssen, Merck, Nektar Therapeutics, Neon Therapeutics, Novartis, Takeda, Tesaro, Transgene, UT Southwestern, Cyteir Therapeutics, Apollomics and Elevation Oncology. M Özgüroğlu and S Murakami have nothing to disclose. A Rydén, Y Zhang and PA Dennis are employees of and have stock ownership in AstraZeneca. C O'Brien is a contracted employee of AstraZeneca. SJ Antonia has received advisory fees from Achilles Therapeutics, Amgen, AstraZeneca, Bristol-Myers Squibb, CBMG, Celsius, GlaxoSmithKline, Memgen, Merck, RAPT, Samyang Biopharma and Venn; travel, accommodations and expenses from Achilles Therapeutics, Amgen, Bristol-Myers Squibb, Celsius, GlaxoSmithKline, Merck and RAPT; and institutional research support from Novartis. The authors have no other relevant affiliations or financial involvement with any organization or entity with a financial interest in or financial conflict with the subject matter or materials discussed in the manuscript apart from those disclosed.

Medical writing support, which was in accordance with Good Publication Practice (GPP3) guidelines, was provided by J Holland and A Korpal of Cirrus Communications (Macclesfield, UK), an Ashfield company, and was funded by AstraZeneca.

Ethical conduct of research

The PACIFIC study protocol and amendments were approved by the relevant ethics committees and the study was performed in accordance with the International Conference on Harmonisation Guidelines on Good Clinical Practice and the Declaration of Helsinki. PRO analyses were performed using EORTC QLQ-C30 and EORTC QLQ-LC13 questionnaires.

\section{Data sharing statement}

The authors certify that this manuscript reports original clinical trial data: NCT02125461. AstraZeneca will meet or exceed data availability as per the commitments made to the EFPIA Pharma Data Sharing Principles. For details of our timelines, please refer to our disclosure commitment at https://astrazenecagrouptrials.pharmacm.com/ST/Submission/Disclosure. Data underlying the findings described in this manuscript, including deidentified individual participant data (primary and secondary outcomes) along with the study protocol and statistical analysis plan, may be obtained in accordance with AstraZeneca's data sharing policy described at https://astrazenecagrouptrials. pharmacm.com/ST/Submission/Disclosure. Qualified researchers can request access to anonymized individual patient-level data from AstraZeneca group of companies sponsored clinical trials via the request portal. All requests will be evaluated as per the AZ disclosure commitment: https://astrazenecagrouptrials.pharmacm.com/ST/Submission/Disclosure. When a request has been approved AstraZeneca will provide access to the deidentified individual patient-level data in an approved sponsored tool. A signed Data Sharing Agreement (non-negotiable contract for data accessors) must be in place before accessing requested information. Additionally, all users will need to accept the terms and conditions of the SAS MSE to gain access.

\section{Open access}

This work is licensed under the Attribution-NonCommercial-NoDerivatives 4.0 Unported License. To view a copy of this license, visit http://creativecommons.org/licenses/by-nc-nd/4.0/ 
- A prespecified analysis in the intent-to-treat (ITT) population of the Phase III PACIFIC trial (ClinicalTrials.gov: NCT02125461) revealed no detrimental effect of durvalumab, versus placebo, on patient-reported outcomes (PROs; symptoms, functioning, or global health status/quality). Despite demonstrated survival benefit with durvalumab versus placebo, improvements in PROs had not been expected due to the lower symptom burden and better baseline function of this population compared with patients in later treatment settings.

- Herein, we report post-hoc, exploratory analyses from PACIFIC investigating the impact of PD-L1 tumor cell (TC) expression (at both prespecified [25\%] and post-hoc [1\%] TC thresholds) on PROs to improve understanding of the risk/benefit profile of durvalumab across different PD-L1 populations (including patients with unknown PD-L1 status). As a secondary focus, we also examined the impact of prior CRT-related variables on key PROs.

- PROs were assessed using the European Organisation for Research and Treatment of Cancer quality of life questionnaire - core 30 (EORTC QLQ-C30) and the lung cancer-specific EORTC QLQ-LC13.

- Of 713 randomized patients, $63 \%$ provided a tissue sample of sufficient quality and quantity to determine PD-L1 expression; $37 \%$ had unknown PD-L1 status.

- Most PROs remained stable over time across all PD-L1 subgroups, with no clinically meaningful between-arm differences (defined as $a \geq 10$ point difference between change in score [at week 48 relative to baseline]) in key PROs (i.e. PROs considered of primary interest for analyses of the ITT population).

- However, consistent with the ITT population, clinically meaningful improvements were observed across most PD-L1 subgroups for symptoms of dysphagia and alopecia, with similar magnitudes of improvement observed in the durvalumab and placebo arms, likely reflecting recovery from the side effects of prior chemoradiation.

- Time to deterioration and improvement rate for key PROs among the PD-L1 subgroups were also generally similar to those of the ITT population, with overlapping treatment effects and $95 \% \mathrm{Cls}$.

- Likewise, most PROs also remained stable over time across the CRT subgroups, with some limited clinically meaningful differences observed between the treatment arms.

- Overall, the results were generally consistent with those of the ITT population, suggesting that durvalumab (compared with placebo) had no detrimental effect on PROs irrespective of PD-L1 expression (including for patients with PD-L1 TC $<1 \%$ ) or variables related to prior CRT.

\section{References}

Papers of special note have been highlighted as: $\bullet$ of interest

1. Ahn JS, Ahn YC, Kim JH et al. Multinational randomized Phase III trial with or without consolidation chemotherapy using docetaxel and cisplatin after concurrent chemoradiation in inoperable stage III non-small-cell lung cancer: KCSG-LU05-04. J. Clin. Oncol. 33(24), 2660-2666 (2015).

2. Yoon SM, Shaikh T, Hallman M. Therapeutic management options for stage III non-small cell lung cancer. World. J. Clin. Oncol. 8(1), 1-20 (2017).

3. Bradley JD, Hu C, Komaki RR et al. Long-term results of NRG oncology RTOG 0617: standard- versus high-dose chemoradiotherapy with or without cetuximab for unresectable stage III non-small-cell lung cancer. J. Clin. Oncol. 38(7), 706-714 (2020).

4. Lima V, Baldotto C, Barrios C et al. Stage III non-small-cell lung cancer treated with concurrent chemoradiation followed or not by consolidation chemotherapy: a survival analysis from a Brazilian multicentric cohort. J. Glob. Oncol. 4, 1-11 (2018).

5. Antonia SJ, Villegas A, Daniel D et al. Durvalumab after chemoradiotherapy in stage III non-small-cell lung cancer. N. Eng. J. Med. 377(20), 1919-1929 (2017).

- Details progression-free survival results for the intent-to-treat (ITT) population in the PACIFIC trial at the first planned interim analysis.

6. Antonia SJ, Villegas A, Daniel D et al. Overall survival with durvalumab after chemoradiotherapy in stage III NSCLC. N. Eng. J. Med. 379(24), 2342-2350 (2018).

- Details overall survival results for the ITT population in the PACIFIC trial. Also, details efficacy analyses per prespecified and post-hoc tumor PD-L1 expression levels.

7. European Medicines Agency. Durvalumab (Imfinzi). Summary of product characteristics 2018. (2019).

- Details the recommended use of Imfinzi per EMA guidelines, including details of efficacy results by PD-L1 subgroup.

8. Faivre-Finn C, Vicente D, Kurata T et al. LBA49 Durvalumab after chemoradiotherapy in stage III NSCLC: 4-year survival update from the Phase III PACIFIC trial. Ann. Oncol. 31(Suppl. 4), S1142-S1215 (2020).

9. US FDA. IMFINZI (Durvalumab) Label 2018. (2019).

10. Mercieca-Bebber R, King MT, Calvert MJ, Stockler MR, Friedlander M. The importance of patient-reported outcomes in clinical trials and strategies for future optimization. Patient Relat. Outcome Meas. 9, 353-367 (2018).

- Details the reasons for the growing incorporation of patient-reported outcome (PRO) data in clinical trials, and the potential benefits which could be derived. 
11. Kluetz PG, O’Connor DJ, Soltys K. Incorporating the patient experience into regulatory decision making in the USA, Europe, and Canada. Lancet Oncol. 19(5), e267-e274 (2018).

- Details the increasing importance placed upon PRO data by regulatory bodies.

12. Efficace F, Fayers P, Pusic A et al. Quality of patient-reported outcome reporting across cancer randomized controlled trials according to the CONSORT patient-reported outcome extension: a pooled analysis of 557 trials. Cancer 121(18), 3335-3342 (2015).

13. Hui R, Özgüroğlu M, Villegas A et al. Patient-reported outcomes with durvalumab after chemoradiotherapy in stage III, locally advanced, unresectable non-small cell lung cancer: results from the randomised Phase III PACIFIC study. Lancet Oncol. 20(12), 1670-1680 (2019).

- Details PRO data for the ITT population in the PACIFIC trial.

14. Shen X, Zhao B. Efficacy of PD-1 or PD-L1 inhibitors and PD-L1 expression status in cancer: meta-analysis. BMJ 362, k3529 (2018).

15. Borghaei H, Paz-Ares L, Horn L et al. Nivolumab versus docetaxel in advanced nonsquamous non-small-cell lung cancer. N. Eng. J. Med. 373(17), 1627-1639 (2015).

16. Brahmer J, Reckamp KL, Baas P et al. Nivolumab versus docetaxel in advanced squamous-cell non-small-cell lung cancer. N. Eng. J. Med 373(2), 123-135 (2015).

17. Rittmeyer A, Barlesi F, Waterkamp D et al. Atezolizumab versus docetaxel in patients with previously treated non-small-cell lung cancer (OAK): a Phase III, open-label, multicentre randomised controlled trial. Lancet 389(10066), 255-265 (2017).

18. Paz-Ares L, Spira A, Raben D et al. Outcomes with durvalumab by tumour PD-L1 expression in unresectable, stage III non-small-cell lung cancer in the PACIFIC trial. Ann. Oncol. 31(6), 798-806 (2020).

19. Ko EC, Formenti SC. Radiotherapy and checkpoint inhibitors: a winning new combination? Ther. Adv. Med. Oncol. 10, doi: $10.1177 / 1758835918768240$ (2018)

20. Deng L, Liang H, Burnette B et al. Irradiation and anti-PD-L1 treatment synergistically promote antitumor immunity in mice. J. Clin. Invest. 124(2), 687-695 (2014).

21. Dovedi SJ, Adlard AL, Lipowska-Bhalla G et al. Acquired resistance to fractionated radiotherapy can be overcome by concurrent PD-L1 blockade. Cancer Res. 74(19), 5458-5468 (2014).

22. Dovedi SJ, Cheadle EJ, Popple AL et al. Fractionated radiation therapy stimulates antitumor immunity mediated by both resident and infiltrating polyclonal t-cell populations when combined with PD-1 blockade. Clin. Cancer Res. 23(18), 5514-5526 (2017).

23. Chacon JA, Schutsky K, Powell DJ. The impact of chemotherapy, radiation and epigenetic modifiers in cancer cell expression of immune inhibitory and stimulatory molecules and anti-tumor efficacy. Vaccines 4(4), 43 (2016).

24. Formenti SC, Demaria S. Combining radiotherapy and cancer immunotherapy: a paradigm shift. J. Nat. Cancer Inst. 105(4), 256-265 (2013).

25. Jiang W, Chan CK, Weissman IL, Kim BYS, Hahn SM. Immune priming of the tumor microenvironment by radiation. Trends Cancer 2(11), 638-645 (2016).

26. Funaki S, Shintani Y, Kawamura T, Kanzaki R, Minami M, Okumura M. Chemotherapy enhances programmed cell death 1/ligand 1 expression via TGF- $\beta$ induced epithelial mesenchymal transition in non-small cell lung cancer. Oncol. Rep. 38(4), 2277-2284 (2017).

27. Adams DL, Adams DK, He J et al. Sequential tracking of PD-L1 expression and RAD50 induction in circulating tumor and stromal cells of lung cancer patients undergoing radiotherapy. Clin. Cancer Res. 23(19), 5948-5958 (2017).

28. Yoneda K, Kuwata T, Kanayama M et al. Alteration in tumoural PD-L1 expression and stromal CD8-positive tumour-infiltrating lymphocytes after concurrent chemo-radiotherapy for non-small cell lung cancer. Br. J. Cancer 121(6), 490-496 (2019).

29. Faivre-Finn C, Spigel DR, Senan S et al. 1363 O Exploratory analyses of overall survival in PACIFIC. Ann. Oncol. 28 (Suppl. 8), viii488-viii492 (2018).

30. Aaronson NK, Ahmedzai S, Bergman B et al. The european organization for research and treatment of cancer QLQ-C30: a quality-of-life instrument for use in international clinical trials in oncology. J. Natl Cancer Inst. 85(5), 365-376 (1993).

31. Bergman B, Aaronson NK, Ahmedzai S, Kaasa S, Sullivan M. The EORTC QLQ-LC13: a modular supplement to the EORTC core quality of life questionnaire (QLQ-C30) for use in lung cancer clinical trials. EORTC study group on quality of life. Eur. J. Cancer 30A(5), 635-642 (1994).

32. Osoba D, Rodrigues G, Myles J, Zee B, Pater J. Interpreting the significance of changes in health-related quality-of-life scores. J. Clin. Oncol. 16(1), 139-144 (1998).

33. Movsas B, Hu C, Sloan J et al. Quality of life analysis of a radiation dose-escalation study of patients with non-small-cell lung cancer: a secondary analysis of the radiation therapy oncology group 0617 randomized clinical trial. JAMA. Oncol. 2(3), 359-367 (2016).

34. Peters S, Dafni U, Boyer M et al. Position of a panel of international lung cancer experts on the approval decision for use of durvalumab in stage III non-small-cell lung cancer (NSCLC) by the committee for medicinal products for human use (CHMP). Ann. Oncol. 30(2), 161-165 (2019).

35. Shin J, Chung JH, Kim SH et al. Effect of platinum-based chemotherapy on PD-L1 expression on tumor cells in non-small cell lung cancer. Cancer Res. Treat. 51(3), 1086-1097 (2018).

36. Deng L, Liang H, Burnette B et al. Irradiation and anti-PD-L1 treatment synergistically promote antitumor immunity in mice. J. Clin. Invest. 124(2), 687-695 (2014). 